\title{
Regulation of tau's proline rich region by its N-terminal domain
}

Kristen McKibben ${ }^{\mathrm{a}}$ and Elizabeth Rhoades ${ }^{\mathrm{a}, \mathrm{b}, 1}$

${ }^{a}$ Biochemistry and Molecular Biophysics Graduate Group, University of Pennsylvania, PA USA

${ }^{\mathrm{b}}$ Department of Chemistry, University of Pennsylvania, PA USA

${ }^{1}$ To whom correspondence may be addressed. Email: elizabeth.rhoades@sas.upenn.edu

Author contributions: K.M. and E.R. designed research. K.M. performed research and analyzed data; K.M. and E.R. wrote the paper.

The authors declare no conflict of interest.

Classification: Biological Sciences/Biophysics and Computational Biology

Keywords: intrinsically disordered proteins, tau, Alzheimer's disease, microtubules, single molecule fluorescence 


\begin{abstract}
Tau is an intrinsically disordered, microtubule-associated protein with a role in regulating microtubule dynamics. Despite intensive research, the molecular mechanisms of taumediated microtubule polymerization are poorly understood. Here we use single molecule fluorescence to investigate the role of tau's N-terminal domain (NTD) and proline rich region (PRR) in regulating interactions of tau with soluble tubulin. Both fulllength tau isoforms and truncated variants are assayed for their ability to bind soluble tubulin and stimulate microtubule polymerization. We describe a novel role for tau's PRR as an independent tubulin-binding domain with polymerization capacity. In contrast to the relatively weak tubulin interactions distributed throughout the microtubule binding repeats (MTBR), resulting in heterogeneous tau:tubulin complexes, the PRR binds tubulin tightly and stoichiometrically. Moreover, we demonstrate that interactions between the PRR and MTBR are reduced by the NTD through a conserved conformational ensemble. Based on our data, we propose that tau's PRR can serve as a core tubulin-binding domain, while the MTBR enhances polymerization capacity by increasing the local tubulin concentration. The NTD negatively regulates tubulin-binding interactions of both of these domains. This study draws attention to the central role of the PRR in tau function, as well as providing mechanistic insight into tau-mediated polymerization of tubulin.
\end{abstract}

\title{
Significance Statement
}

Tau is an intrinsically disordered, microtubule associated protein linked to a number of neurodegenerative disorders. Here we identify tau's proline rich region as having autonomous tubulin binding and polymerization capacity, which is enhanced by the flanking microtubule binding repeats. Moreover, we demonstrate that tau's N-terminal domain negatively regulates both binding and polymerization. We propose a novel model for tau-mediated polymerization whereby the proline rich region serves as a core tubulinbinding domain, while the microtubule binding repeats increase the local concentration. Our work draws attention to the importance of the proline rich region and N-terminal domain in tau function, and highlights the proline rich region as a putative target for the development of therapeutics. 


\section{Introduction}

Tau belongs to a family of microtubule associated proteins that generally function to modulate microtubule stability and dynamics $(1,2)$. The deposition of aggregates of tau is linked to a number of neurodegenerative disorders, collectively known as tauopathies [reviewed in (3)]. Cell death is thought to arise both from the process of aggregation as well as from the loss of functional tau and subsequent destabilization of microtubules (3-5).

Tau is an intrinsically disordered protein and it appears to remain largely disordered even upon binding to soluble tubulin (6) or microtubules $(7,8)$. In vitro, tau decreases the critical concentration for tubulin polymerization and regulates microtubule growth rates, catastrophe frequency and recovery (9-11). More recently, tau has been observed to sequester soluble tubulin during liquid-liquid phase separation, leading to microtubule polymerization and bundling (12). It was proposed that this phenomenon may underlie the initiation of microtubules in the axons of neurons.

In the brain, there are six different isoforms of tau, arising from alternative splicing, resulting in the presence of 0,1 or 2 inserts in the N-terminal domain (NTD) and 3 or 4 repeats within the microtubule binding region (MTBR) (Fig. 1) (13). These isoforms are developmentally regulated with varying distributions of isoforms across developmental stage, cell type and cellular location $(3,13)$. The microtubule binding region (MTBR) is the best-studied region of tau, both because it contains the 31 or 32 residue long eponymous repeat sequences that are important for binding to microtubules $(14,15)$, but is also forms the core of aggregates in tauopathies (Fig. 1) (16-18). More recent studies have focused on the role of $\mathrm{R}^{\prime}$, the $\sim 25$ residues C-terminal to the MTBR, a highly conserved sequence sometimes referred to as a pseudo-repeat (Fig. 1) (19-22). Nterminally flanking the MTBR is the proline rich region (PRR) composed of $\sim 25 \%$ prolines across two sub-regions, P1 and P2 (Fig. 1). The addition of P2 and R' to MTBR fragments increases microtubule binding and stimulates polymerization $(9-11,19,20,22$, 23). The NTD together with P1 is thought to regulate binding to and spacing of microtubules $(9,24-27)$, and mediate interactions with other cellular partners such as signaling proteins [reviewed in (28)] or the plasma membrane (29). 
Despite intense interest in tau, the molecular details of its numerous proposed functions remain relatively obscure. This is in part due to the challenges that arise from its lack of stable structure (30), and that it does not seem to form well-defined stoichiometric complexes with tubulin (20). To illustrate, it was demonstrated more than 20 years ago that P2 $(9,10,22)$ greatly enhanced tau binding to microtubules and its ability to polymerize tubulin (31), yet this region of tau is not observed in a recent structure of microtubule-bound tau (7). It may be that the PRR enhances binding indirectly through interactions with the MTBR (22), or that bound PRR is too disordered and dynamic on the microtubule surface to be resolved by EM. These apparently diverging observations, and the need to reconcile them, highlights the requirement for studies of tau function that look beyond the MTBR.

Here, we investigate the role of the NTD and PRR in regulating tau's interactions with soluble tubulin. Single molecule Förster resonance energy transfer (smFRET) and fluorescence correlation spectroscopy (FCS) of the full-length, N-terminal variant isoforms that contains four MTBR repeats $\left(\operatorname{tau}_{2 \mathrm{~N}}, \operatorname{tau}_{1 \mathrm{~N}}\right.$, and tau $\left.\mathrm{u}_{0 \mathrm{~N}}\right)$ were used to monitor binding and probe tau's conformation in tau:tubulin assembles. We found that in the absence of tubulin the NTD interacts with the PRR and MTBR through a conserved conformational ensemble. The NTD negatively regulates binding to soluble tubulin and subsequently slows polymerization. Strikingly, we find that the isolated PRR is capable of both stoichiometric binding to, and polymerization of, soluble tubulin. The presence of the NTD dramatically reduces the binding and polymerization capacity of the PRR. Based on our results, we propose a model where the PRR serves as a core tubulin binding domain of tau, with both binding and polymerization capacity enhanced by the MTBR and R', and reduced by the NTD.

\section{Results}

Conformational ensemble of tau's NTD/PRR/MTBR is conserved across isoforms.

In solution, the N-terminus of tau makes relatively close contacts with both the MTBR and the C-terminus (32), which are lost when tau binds soluble tubulin (6). We used smFRET to assess how the N-terminal inserts impact tau's solution conformational ensemble. Full-length tau isoforms were labeled with donor and acceptor fluorophores at 
sites spanning domains of interest (Fig. 2A). The mean energy transfer efficiencies, $\mathrm{ET}_{\text {eff, }}$ were converted to experimental root-mean-square $\left(\mathrm{RMS}_{\mathrm{exp}}\right)$ distances using a Gaussian coil model (see SI Appendix for details). For constructs probing the C-terminus, tau ${ }^{291-433}$, as well as the PRR, tau ${ }^{149-244}$, all three isoforms gave rise to comparable $\mathrm{RMS}_{\text {exp }}$ values (Figs. 2B,C and S1B,C and Table S2); this was expected, as the number of residues encompassed by the probes is the same for all three isoforms. The constructs probing the N-terminal domain (NTD), tau ${ }^{17-149}$, also exhibited predicted behavior in that the presence of each N-terminal insert resulted in an increase in the $\mathrm{RMS}_{\exp }$ (Fig. 2B,C and Table S2). Interestingly, constructs whose labels span the NTD through the PRR, tau ${ }^{17-}$ ${ }^{244}$, or the NTD through part of the MTBR, tau ${ }^{17-291}$ had comparable ET $_{\text {eff }}$ histograms, and thus $\mathrm{RMS}_{\text {exp }}$ values, in solution, despite an increase of up to 60 residues between isoforms (Figs. 2B,C and S1B,C and Table S2). The similar inter-domain distances suggest homologous conformational ensembles between isoforms. Moreover, these ensembles are relatively compact. To illustrate, the $\mathrm{RMS}_{\exp }$ values of the tau ${ }^{17-291}$ isoforms were of similar magnitude to those of tau ${ }^{149-244}$ despite being 120 to 180 residues longer (Table S2). The $\mathrm{RMS}_{\text {exp }}$ values for the constructs probing the entirety of the isoforms, $\operatorname{tau}^{17-433}$, were also nearly equivalent, consistent with the tau ${ }^{17-291}$ and tau ${ }^{291-}$ ${ }^{433} \mathrm{RMS}_{\text {exp }}$ values reported here (Figs. 2B,C and S1B,C and Table S2). Upon binding to tubulin, deviations from scaling behavior were diminished, and all constructs yielded $\mathrm{RMS}_{\text {exp }}$ values that scale with the number of residues in a manner consistent with an extended, random structure (Fig. S1C) $(6,7,33)$.

\section{Tau's NTD negatively regulates tubulin binding.}

The conservation of the conformational ensembles across N-terminal isoforms suggests a functional origin. This led us to examine the impact of the N-terminal inserts on tau binding to soluble tubulin. Tubulin binding of fluorescently labeled tau in the presence of increasing concentrations of tubulin was assessed by FCS under nonpolymerizing conditions. Both the longest full-length isoform, tau $2 \mathrm{~N}$, and an NTD deletion fragment, tau $\mathrm{u}_{\Delta \mathrm{N}}$ (amino acids 149 to 441), bound tubulin as seen by an increase in the normalized diffusion time, $\tau_{\text {norm, }}$ with increasing tubulin concentration (Fig. $3 \mathrm{~A}$ ). However, there are significant differences in the binding curves; tau $2 \mathrm{~N}$ reached its 
maximum $\tau_{\text {norm }}$ at $\sim 2.5 \mu \mathrm{M}$ tubulin, while the $\tau_{\text {norm }}$ for tau $u_{\Delta \mathrm{N}}$ continued to increase. At 10 $\mu \mathrm{M}$ tubulin, the $\tau_{\text {norm }}$ of $\operatorname{tau}_{\Delta \mathrm{N}}$ was more than $2 \mathrm{x}$ larger than that of $\operatorname{tau}_{2 \mathrm{~N}}($ Fig. 3A). This effect was specific to the NTD. Binding by a C-terminal deletion construct, $\operatorname{tau}_{\Delta \mathrm{C}}(\operatorname{amino}$ acids 1 to 395), resembled that of tau $\mathrm{u}_{\mathrm{N}}$ (Fig. 3A) while a combined N-terminal and Cterminal deletion construct, PRR-MTBR-R' (amino acids 149 to 395) behaved like tau ${ }_{\Delta \mathrm{N}}$, (Fig. 3A). These measurements suggest that the NTD of tau reduces or negatively regulates its binding to soluble tubulin, while the $\mathrm{C}$-terminus does not have a significant role. Finally, we measured the tubulin binding by all three N-terminal isoform variants, $\operatorname{tau}_{2 \mathrm{~N}}, \operatorname{tau}_{1 \mathrm{~N}}$ and $\mathrm{tau}_{0 \mathrm{~N}}$, and found their binding curves to be comparable (Fig. S2A), indicating that regulation of binding is intrinsic to the NTD and not strongly dependent on the presence or absence of a specific insert.

In our prior work, we demonstrated tau forms fuzzy complexes with soluble tubulin consisting of multiple, weakly-associated tubulin dimers (20). Using a similar approach as described in that work, we analyzed the individual autocorrelation curves taken in the presence of $10 \mu \mathrm{M}$ tubulin in order to assess the heterogeneity of the tautubulin complexes (Fig. 3B; details of analysis are in the SI Appendix). From this analysis, it was apparent that not only were tau:tubulin complexes formed by PRRMTBR-R' on average larger (median diffusion time, $\tau_{\text {median }}=2.02 \mathrm{~ms}$ ) than those formed by any of the full-length constructs $\left(\tau_{\text {median }}=1.29,1.50\right.$, and $1.55 \mathrm{~ms}$ for tau ${ }_{2 \mathrm{~N}}, \operatorname{tau}_{1 \mathrm{~N}}$ and $\operatorname{tau}_{0 \mathrm{~N}}$, respectively), but that PRR-MTBR-R':tubulin complexes also had the largest spread in diffusion times (1.26 to $2.89 \mathrm{~ms}$ ). These complexes persisted at $300 \mathrm{mM} \mathrm{KCl}$, indicating they were not the result of low salt buffer (Table S5). Analysis of the average brightness of the diffusing species demonstrated that while the full-length constructs typically consisted of a single tau molecule, the PRR-MTBR-R':tubulin complexes, especially the larger ones, may have included several tau molecules (Fig. S2B,C and Table S6). Together, analysis of diffusion time and brightness of the complexes suggest that the NTD limits both: (1) the average number of tubulin dimers bound to a single tau molecule; and (2) the average number of tau bound to a single tubulin dimer.

The prior work from our lab also demonstrated a positive correlation between the rate of tau-mediated tubulin polymerization and the median diffusion time, and thus the number of tubulin dimers associated, of tau:tubulin complexes (20). That work, however, 
exclusively used fragments of tau, none of which included the entire PRR or the NTD. Nevertheless, we found that this observation broadly holds for the full-length and fragments studied here: PRR-MTBR-R' had the fastest polymerization half-time $\left(t_{1 / 2}=52\right.$ $\pm 7 \mathrm{~s}$ ) while the full-length isoforms were all slower (Fig. 3C). Interestingly, tau $2 \mathrm{~N}$ was the slowest $\left(t_{1 / 2}=137 \pm 9 \mathrm{~s}\right)$, lagging behind $\operatorname{tau}_{1 \mathrm{~N}}$ and $\operatorname{tau}_{0 \mathrm{~N}}\left(t_{1 / 2}=88 \pm 13 \mathrm{~s}\right.$ and $76 \pm 10 \mathrm{~s}$, respectively) (Fig. 3C and Table S4). This, along with the small decrease in the size and heterogeneity of the tubulin complexes formed by tau ${ }_{2 \mathrm{~N}}$ (Fig. 3A), suggests that the N2 insert may have an additional regulatory role in binding and polymerizing tubulin.

\section{The PRR independently binds and polymerizes tubulin}

The reduced binding of NTD containing constructs (Fig. 3) coupled with the conserved conformational ensembles in the NTD/PRR/MTBR constructs observed in the smFRET measurements (Figs. 2 and S1), led us to hypothesize that the NTD may regulate tubulin binding though interactions with the PRR or MTBR. To investigate this hypothesis, we created constructs corresponding to these domains and measured binding by FCS as well as tau-mediated polymerization. Although the MTBR (amino acids 244 to 372) associates with microtubules in the context of the full-length protein or in constructs containing P2 $(7,15)$, the isolated domain bound only weakly to soluble tubulin (Fig. 4A) and was not capable of polymerizing tubulin (Fig. 4B). The addition of R', MTBR-R' (amino acids 244-395), enhanced binding (Fig. 4A) but still did not yield a construct that promoted efficient polymerization (Fig. 4B). Although early studies demonstrated that the MTBR-R' (9) or even peptides corresponding to the individual MTBR repeats (34) had weak polymerization capacity, 5 to 10 -fold more tau was required than the $10 \mu \mathrm{M}$ used here.

Strikingly - and surprisingly - the isolated PRR (amino acids 149 to 244), bound tubulin tightly when compared to the MTBR and MTBR-R' measured under the same conditions (Fig. 4A). It also stimulated rapid polymerization of tubulin (Fig. 4B). Fitting the PRR:tubulin binding curve to the Hill equation yields $n=1.7 \pm 0.2$, reflecting cooperativity, and with an apparent $\mathrm{K}_{\mathrm{D}} \approx 900 \mathrm{nM}$ (Fig. S3). Furthermore, unlike constructs where the PRR is coupled with the MTBR and/or R', such as PRR-MTBR or PRR-MTBR-R', the PRR demonstrated saturable binding and did not form large 
tau:tubulin complexes (Fig. S4). Based on saturation diffusion time measured for PRR:tubulin at $10 \mu \mathrm{M}$ tubulin, we estimate there to be $\sim 2$ binding sites for tubulin dimers in the PRR (see SI Appendix). This apparent specificity suggests that formation of tau:tubulin fuzzy complexes arises primarily from the collective binding properties of the PRR and MTBR-R'.

While the PRR and R' have been identified as enhancing binding and accelerating polymerization in vitro $(9-11,19,20,22,23,35,36)$ these features have not previously been observed for the isolated PRR. This may in large part be due to the widespread use of the K16 fragment consisting of P2 and the 4R MTBR (amino acids 198 to 372, P2MTBR) (9). The P2-MTBR construct binds to tubulin, however, it does not bind as many tubulin dimers at high tubulin concentrations as PRR-MTBR (Fig. S5). Thus, while the isolated P1 does not bind tubulin strongly (Fig. S6), it does enhance binding and contribute to tau function. Tight binding to tubulin required the presence of both proline rich regions; fragments corresponding to P1 (amino acids 149 to 198) or P2 (amino acids 199 to 244) bound tubulin only weakly (Fig. S6).

\section{NTD negatively regulates the polymerization capacity of the PRR}

Our observation that the PRR bound to and polymerized tubulin independently of the MTBR (Fig. 4), combined with the slower polymerization rate of tau constructs including the NTD $\left(\operatorname{tau}_{2 \mathrm{~N}}, \operatorname{tau}_{1 \mathrm{~N}}\right.$ and tau $\left.\mathrm{uN}_{0 \mathrm{~N}}\right)$ relative to PRR-MTBR-R', motivated us to determine the impact of the NTD on interactions of the PRR with tubulin. $\mathrm{Tau}_{2 \mathrm{~N}}$ was truncated after the PRR at amino acid 244 (2N-PRR), and binding to soluble tubulin and polymerization were measured. The presence of the NTD dramatically reduced binding (Fig. 4A) as well as significantly diminished tubulin polymerization capability (Fig. 4B). Truncated constructs based on tau $\mathrm{u}_{1 \mathrm{~N}}$ and $\operatorname{tau}_{0 \mathrm{~N}}, 1 \mathrm{~N}-\mathrm{PRR}$ and $0 \mathrm{~N}-\mathrm{PRR}$ respectively, showed similar binding behavior (Fig. S7). Collectively, these results led us to propose that the binding and - by extension - polymerization capacity of tau is regulated by interactions between the NTD and the PRR, evident by the conserved ensembles observed with smFRET for this domain (tau ${ }^{17-244}$ in Fig. 2). Because the conserved ensembles extend into the MTBR (tau ${ }^{17-291}$ in Figs. 2 and S1), we tested this idea by making a construct lacking the PRR (2N-MTBR-R' amino acids 1 to 148 fused to 245 to 
395). This construct also did not demonstrate appreciable binding to tubulin (Fig. 4A), while that same construct lacking the NTD (MTBR-R') clearly did (Fig. 4A). As a whole, these results strongly support a functional, regulatory role for the compact, albeit disordered, NTD/PRR/MTBR ensembles observed by smFRET.

\section{Discussion}

Since it was first isolated over 40 years ago (1), both functional and structural studies of tau have primarily focused on the MTBR $(7-9,14,37)$. Our current study examines two domains of tau that have been the subject of significantly less scrutiny: the NTD and the PRR. Our discovery that the isolated PRR has the capacity to bind tubulin and polymerize microtubules in vitro, and that this function is negatively regulated by the NTD, draws attention to the importance of these two domains in understanding tau function.

The NTD has previously been shown to regulate in vitro polymerization of tubulin (9). It is implicated in isoform-dependent spacing of microtubules $(24,25)$, and removal of the NTD both increases the affinity for microtubules as well as results in the presence of large microtubule bundles (9). Here we observed a similar inhibitory effect in binding to soluble tubulin (Fig. 3A). This inhibition seems to be an effect of the NTD as a whole, rather than resulting from the absence or presence of a specific insert, as only small differences in binding are observed for the $0 \mathrm{~N}, 1 \mathrm{~N}$ and $2 \mathrm{~N}$ isoforms in comparison to when the NTD is absent (Fig. 3B). Insight into why the inserts do not have a significant effect in regulating binding is gained from our smFRET measurements which show that the relative dimensions corresponding to the NTD-PRR ( $\operatorname{tau}^{17-244}$ ) or NTDMTBR ( $\operatorname{tau}^{17-291}$ ) are independent of the number of inserts (Fig. 2 and S1). This suggests that conserved long-range interactions and/or conformational features of the NTD are important for regulating interactions with tubulin, more so than the inserts themselves. Given that the NTD also significantly reduces the size and heterogeneity of 'fuzzy' tautubulin complexes (Fig. 3) (20), it follows that the NTD may dynamically shield the weak tubulin binding sites distributed throughout the MTBR and R' (Fig. 5). Our results suggest this screening is a general function of the NTD that serves as an initial regulatory gate to tau-mediated polymerization, and is largely independent of the individual $\mathrm{N}$ - 
terminal inserts. These results also indicate that the regulatory mechanism of the NTD for tau:tubulin is distinct from that of tau:microtubules, where the relative electrostatics and sterics of different $\mathrm{N}$-terminal isoforms impact the tau:tau interactions required for spacing and bundling of microtubules $(9,24-27)$.

Our observation of binding to and assembly of tubulin by the isolated PRR was unexpected, as to our knowledge, there are no prior reports of this in the literature. NMR chemical shifts suggesting binding were measured in the PRR of longer tau fragments in the presence of engineering tubulin constructs $(8,11)$. For a tau fragment covering part of the PRR, residues 166-244, 1:2 tau:tubulin dimer stoichiometry was observed with taxol stabilized microtubules, but polymerization was not tested (11). We also find tight, saturable stoichiometric binding of 1:2 tau:tubulin dimers. Notably, PRR residues were not observed in the recent cryo-EM structure of microtubule-bound tau (7). It may be that the PRR binds to a region unresolved within the structure, such as intrinsically disordered loops or the tubulin tails, or to a site partially occluded in the microtubule lattice.

The stoichiometric binding to soluble tubulin of the PRR provides a striking contrast with the dynamic, heterogeneous 'fuzzy' tau-tubulin complexes formed when the MTBR and $\mathrm{R}^{\prime}$ were present in the constructs. In particular, tight and specific binding of the PRR may offer an attractive target for drug development relative to the comparatively weak binding by the MTBR-R' (38). Interestingly, both P2 and R' were identified relatively early as sequences important for productive tau-mediated polymerization (39). The 'jaws' model proposed that targeting of tau to the MT lattice is through these regions, while the MTBR played a catalytic role in assembly $(10,19,39)$. However, in our study, the PRR is the only isolated domain which demonstrates any significant tubulin polymerization capacity; this is not seen for MTBR nor MTBR-R' (Fig. 4). This leads us to propose a variation to that model (Fig. 5). In our model, the PRR serves as the core tubulin binding domain, binding to two tubulin dimers in a critical step towards initiating polymerization. Multiple weak tubulin binding sites in the MTBR and R' allow for increasing the local concentration of tubulin, resulting in accelerated microtubule growth. The ubiquitous screening by the NTD of both the PRR and the MTBR serves as an initial gating that controls the size of these tau:tubulin ensembles and, consequently, tubulin assembly. 
Tau's interactions with microtubules are regulated by phosphorylation [reviewed in (40) and (41)] and the majority of tau's 20+ known phosphorylation sites are located in the PRR, including those associated with Alzheimer's disease $(42,43)$. Given this, perhaps the relative importance of the PRR in both binding to and polymerizing tubulin should not be so surprising. In contrast, the MTBR is primarily modified by lysine acetylation. Individual or combinatorial effects of these modifications may alter both the binding affinity and the stoichiometry of tubulin binding. There is a least one example of coordinated modifications to tau in the literature: acetylation at residues 280/281 within the MTBR influences phosphorylation at residues S202/205 within the PRR (44). Either of these modifications may in turn influence the interaction of the PRR or MTBR with the NTD, suggesting that regulation of binding may be more complex than simply reducing affinity and stoichiometry, but instead a intricate interplay between the NTD, PRR and MTBR domains.

\section{Material and Methods}

Protein expression and labeling. Tubulin was purified from fresh bovine brains as described previously (45). Tau constructs were cloned using site-directed mutagenesis or deletion cloning techniques, and purified as described previously (46). Site-specific labeling for FCS and FRET measurements were though introduced cysteines reacted with Alexa Fluor 448 or Alexa Fluor 594 maleimide. See SI Appendix for details.

smFRET and FCS measurements. SmFRET and FCS measurements were carried out on a home-built instrument based on an inverted Olympus $1 \mathrm{X}-71$ microscope $(20,46)$ or a commercial MicroTime 200 time-resolved confocal microscope (Picoquant). For smFRET, 20-40 pM tau was used; for FCS, 15-25 nM tau was used. Measurements were made in the absence or presence of tubulin at $20^{\circ} \mathrm{C}$ under non-assembly conditions in phosphate buffer $(20 \mathrm{mM}$ potassium phosphate $\mathrm{pH} 7.4,20 \mathrm{mM} \mathrm{KCl}, 1 \mathrm{mM} \mathrm{MgCl}, 0.5$ $\mathrm{mM}$ EGTA, $1 \mathrm{mM}$ DTT). For smFRET, the $\mathrm{ET}_{\text {eff }}$ was calculated from photon burst intensities and converted to $\mathrm{RMS}_{\text {exp }}$ distances using a Gaussian polymer chain model (47). For FCS, multiple autocorrelation curves were collected and fit using a singlecomponent 3D diffusion model (20). For heterogeneity analysis, curves were assessed for 
goodness-of-fit and then filtered until a stable population was reached using iterative filtering of diffusion times. See SI Appendix for details.

Tubulin polymerization. Clarified tubulin was incubated with tau at varying ratios at $4{ }^{\circ} \mathrm{C}$ prior to the addition of $1 \mathrm{mM}$ GTP and immediate transfer to phosphate buffer in a cuvette equilibrated to $37{ }^{\circ} \mathrm{C}$. Polymerization was monitored by an increase in the scattered light signal. Normalized curves were fit to a sigmoidal growth equation, Eq. S3. See SI Appendix for details.

Acknowledgements. We would like to thank S. Wickramasinghe, H.Y.J Fung, I. Yannatos and H. Merens for assistance during the various tubulin purifications, and D. Chenoweth for use of his fluorimeter. This work was supported by NIH Institutional Training Grant T32 GM071399 (to K.M.) and NIH Grant AG053951 (to E.R.).

\section{References}

1. Weingarten MD, Lockwood AH, Hwo SY, Kirschner MW (1975) A protein factor essential for microtubule assembly. Proc Natl Acad Sci USA 72:1858-1862.

2. Drubin DG, Kirschner MW (1986) Tau protein function in living cells. J Cell Biol 103:2739-2746.

3. Guo T, Noble W, Hanger DP (2017) Roles of tau protein in health and disease. Acta Neuropath 133:665-704.

4. Ballatore C, Lee VMY, Trojanowski JQ (2007) Tau-mediated neurodegeneration in alzheimer's disease and related disorders. Nature Rev Neurosci 8:663-672.

5. Winklhofer KF, Tatzelt J, Haass C (2008) The two faces of protein misfolding: Gain- and loss-of-function in neurodegenerative diseases. EMBO J 27:336-349.

6. Melo AM, et al. (2016) A functional role for intrinsic disorder in the tau-tubulin complex. Proc Natl Acad Sci USA 113:14336-14341.

7. Kellogg EH, et al. (2018) Near-atomic model of microtubule-tau interactions. Science 360:1242-1246.

8. Mukrasch MD, et al. (2009) Structural polymorphism of 441-residue tau at single residue resolution. PLoS Biol 7:e34.

9. Gustke N, Trinczek B, Biernat J, Mandelkow E (1994) Domains of tau protein and interactions with microtubules. Biochemistry 32:9511-9522.

10. Trinczek B, Biernat J, Baumann K, Mandelkow EM, Mandelkow E (1995) Domains of tau protein, differential phosphorylation, and dynamic instability of microtubules. Mol Biol Cell 6:1887-1902.

11. Fauquant C, et al. (2011) Systematic identification of tubulin interacting fragments of the microtubule-associated protein tau leads to a highly efficient promoter of microtubule assembly. J Biol Chem 286:33358-33368 
12. Hernández-Vega A, et al. (2017) Local nucleation of microtubule bundles through tubulin concentration into a condensed tau phase. Cell Rep 20:2304-2312.

13. Andreadis A (2005) Tau gene alternative splicing: Expression patterns, regulation and modulation of function in normal brain and neurodegenerative diseases. Biochim Biophys Acta 1739:91-103.

14. Goode BL, Chau M, Denis PE, Feinstein SC (2000) Structural and functional differences between 3-repeat and 4-repeat tau isoforms: Implications for normal tau function and the onset of neurodegenerative disease. J Biol Chem 275:3818238189.

15. Butner KA, Kirschner MW (1991) Tau protein binds to microtubules through a flexible array of distributed weak sites. J Cell Biol 115:717-730.

16. Falcon B, et al. (2019) Novel tau filament fold in chronic traumatic encephalopathy encloses hydrophobic molecules. Nature 568:420-423.

17. Fitzpatrick AWP, et al. (2017) Cryo-em structures of tau filaments from alzheimer's disease. Nature 547:185-190.

18. Falcon B, et al. (2018) Structures of filaments from pick's disease reveal a novel tau protein fold. Nature 561:137-140.

19. Mukrasch MD, et al. (2007) The "jaws" of the tau-microtubule interaction. J Biol Chem 282:12230-12239.

20. Li X-H, Rhoades E (2017) Heterogeneous tau-tubulin complexes accelerate microtubule polymerization. Biophys $J$ 112:2567-2574.

21. Niewidok B, et al. (2016) Presence of a carboxy-terminal pseudorepeat and disease-like pseudohyperphosphorylation critically influence tau's interaction with microtubules in axon-like processes. Mol Biol Cell 27:3537-3549.

22. Goode BL, et al. (1997) Functional interactions between the proline-rich and repeat regions of tau enhance microtubule binding and assembly. Mol Biol Cell 8:353-365.

23. Gigant B, et al. (2014) Mechanism of tau-promoted microtubule assembly as probed by nmr spectroscopy. J Am Chem Soc 136:12615-12623.

24. Rosenberg KJ, Ross JL, Feinstein HE, Feinstein SC, Israelachvili J (2008) Complementary dimerization of microtubule-associated tau protein: Implications for microtubule bundling and tau-mediated pathogenesis. Proc Natl Acad Sci USA 105:7445-7450.

25. Chung PJ, et al. (2015) Direct force measurements reveal that protein tau confers short-range attractions and isoform-dependent steric stabilization to microtubules. Proc Natl Acad Sci U S A 112:E6416-6425.

26. Chen J, Kanai Y, Cowan NJ, Hirokawa N (1992) Projection domains of map2 and tau determine spacings between microtubules in dendrites and axons. Nature 360:674-677.

27. Scott CW, Klika AB, Lo MMS, Norris TE, Caputo CB (1992) Tau protein induces bundling of microtubules in vitro: Comparison of different tau isoforms and a tau protein fragment. $J$ Neurosci Res 33:19-29.

28. Morris M, Maeda S, Vossel K, Mucke L (2011) The many faces of tau. Neuron 70:410-426. 
29. Brandt R, Léger J, Lee G (1995) Interaction of tau with the neural plasma membrane mediated by tau's amino-terminal projection domain. J Cell Biol 131:1327-1340.

30. Cleveland DW, Hwo S-Y, Kirschner MW (1977) Physical and chemical properties of purified tau factor and the role of tau in microtubule assembly. $J$ Mol Biol 116:227-247.

31. Goode B, Feinstein S (1994) Identification of a novel microtubule binding and assembly domain in the developmentally regulated inter-repeat region of tau. $J$ Cell Biol 124:769-782.

32. Elbaum-Garfinkle S, Rhoades E (2012) Identification of an aggregation-prone structure of tau. J Am Chem Soc 134:16607-16613.

33. Mukrasch MD, et al. (2009) Structural polymorphism of 441-residue tau at single residue resolution. PLOS Biology 7:e1000034.

34. Ennulat DJ, Liem RK, Hashim GA, Shelanski ML (1989) Two separate 18-amino acid domains of tau promote the polymerization of tubulin. J Biol Chem 264:5327-5330.

35. Farías R GA, Vial C, Maccioni R (1992) Specific macromolecular interactions between tau and the microtubule system. 112:81-88.

36. Mukrasch MD, et al. (2007) The "jaws" of the tau-microtubule interaction. J Biol Chem 282:12230-12239.

37. Maccioni RB, Rivas CI, Vera JC (1988) Differential interaction of synthetic peptides from the carboxyl-terminal regulatory domain of tubulin with microtubule-associated proteins. EMBO J 7:1957-1963.

38. Chong FP, Ng KY, Koh RY, Chye SM (2018) Tau proteins and tauopathies in alzheimer's disease. Cell Mol Neurobiol 38:965-980.

39. Preuss U, Biernat J, Mandelkow EM, Mandelkow E (1997) The 'jaws' model of tau-microtubule interaction examined in CHO cells. J Cell Sci 110:789-800.

40. Hanger DP, Anderton BH, Noble W (2009) Tau phosphorylation: The therapeutic challenge for neurodegenerative disease. Trends Mol Med 15:112-119.

41. Buée L, Bussière T, Buée-Scherrer V, Delacourte A, Hof PR (2000) Tau protein isoforms, phosphorylation and role in neurodegenerative disorders. Brain Res Rev 33:95-130.

42. Morris M, et al. (2015) Tau post-translational modifications in wild-type and human amyloid precursor protein transgenic mice. Nat Neurosci 18:1183-1189.

43. Funk KE, et al. (2014) Lysine methylation is an endogenous post-translational modification of tau protein in human brain and a modulator of aggregation propensity. Biochem J 462:77-88.

44. Trzeciakiewicz H, et al. (2017) A dual pathogenic mechanism links tau acetylation to sporadic tauopathy. Scientific Reports 7:44102-44102.

45. Castoldi M, Popov AV (2003) Purification of brain tubulin through two cycles of polymerization-depolymerization in a high-molarity buffer. Protein Expr Purif 32:83-88.

46. Elbaum-Garfinkle S, Cobb G, Compton JT, Li X-H, Rhoades E (2014) Tau mutants bind tubulin heterodimers with enhanced affinity. Proc Natl Acad Sci USA 111:6311-6316. 
47. O'Brien EP, Morrison G, Brooks BR, Thirumalai D (2009) How accurate are polymer models in the analysis of förster resonance energy transfer experiments on proteins? The Journal of Chemical Physics 130:124903.

48. Kohn JE, et al. (2004) Random-coil behavior and the dimensions of chemically unfolded proteins. Proc Natl Acad Sci USA 101:12491-12496.

\section{Figure Legends}

Fig. 1. Schematics of tau constructs. The uppermost schematic is the longest tau isoform, $\operatorname{tau}_{2 \mathrm{~N}}$. The domains and corresponding residues that delineate them are marked: $\mathrm{N}$ terminal domain (NTD) with N-terminal inserts (N1, N2), proline-rich region (PRR) with sub-regions (P1, P2), microtubule binding repeats (MTBR) with four imperfect repeat sequences (R1-R4) flanked by the pseudo-repeat R' and the C-terminus. Below are the additional tau isoforms and truncated variants used in this study. All numbering of residues throughout the manuscript is based on $\operatorname{tau}_{2 \mathrm{~N}}$.

Fig. 2. SmFRET of tau N-terminal isoforms. (A) Schematic of reference construct tau $2 \mathrm{~N}$ with residues labeled for smFRET measurements are indicated. (B) SmFRET histograms of $\operatorname{tau}_{2 \mathrm{~N}}, \operatorname{tau}_{1 \mathrm{~N}}$ and tau $0 \mathrm{~N}$ labeled at residues 291 and 433 (left), 17 and 149 (center), 17 and 244 (right) in the absence (dark: left axis) and presence (light: right axis) of $10 \mu \mathrm{M}$ tubulin. Histograms for tau labeled at residues 17 and 433, 17 and 291, 149 and 244 are in Fig. S1. Fits to the data as a sum of Gaussian distributions as described in the $S I$ Appendix are shown. (C) For measurements in the absence of tubulin, the root-meansquare distances (RMS) between labeling positions calculated from a Gaussian chain model $\left(\mathrm{RMS}_{\text {exp }}\right)$ are plotted (47). Data are presented as mean $\pm \mathrm{SD}, \mathrm{n} \geq 3$ independent measurements. For reference, the RMS calculated for a random coil $\left(\mathrm{RMS}_{\mathrm{RC}}\right)$ as in Reference (48) is indicated by the gray dashed line. See Table S2 for numerical values of $\mathrm{ET}_{\mathrm{eff}}$ mean $\pm \mathrm{SD}, \mathrm{RMS}_{\mathrm{exp}} \pm \mathrm{SD}$ and $\mathrm{RMS}_{\mathrm{RC}}$ for each construct.

Fig. 3. Inhibition of binding and polymerization by the NTD. (A) The increase in the normalized diffusion time, $\tau_{\text {norm, }}$, as a function of tubulin concentration reflects binding of fluorescently labeled tau to unlabeled tubulin. Data are presented as mean $\pm \mathrm{SD}, \mathrm{n} \geq 3$ independent measurements. See SI Appendix for details of data analysis. See Table S5 for 
numerical values for $\tau_{\mathrm{D}}$ and $\tau_{\text {norm }}$ at $10 \mu \mathrm{M}$ tubulin. (B) The autocorrelation curves for $\operatorname{tau}_{2 \mathrm{~N}}, \operatorname{tau}_{1 \mathrm{~N}}, \operatorname{tau}_{0 \mathrm{~N}}$ and PRR-MTBR-R' in the presence of $10 \mu \mathrm{M}$ tubulin were individually fit to obtain a distribution of $\tau_{\text {norm }}$ values. When the NTD is absent, larger tau-tubulin complexes form as seen by the larger values of $\tau_{\text {norm. Overlays are lognormal }}$ distributions. See Table S3 for descriptive statistics of distributions. (C) Tubulin polymerization as measured by scattered light at $340 \mathrm{~nm}$ as a function of time. See Table $\mathrm{S} 4$ for fit parameters. Data are presented as mean $\pm \mathrm{SD}$ following normalization, $\mathrm{n}=3$ independent measurements. Arrows indicate depolymerization at $4{ }^{\circ} \mathrm{C}$.

Fig. 4. Independent polymerization capacity of the PRR, regulated by the NTD. (A) Binding of tau constructs to tubulin as measured by an increase in $\tau_{\text {norm }}$ as a function of tubulin concentration. Data are presented as mean $\pm \mathrm{SD}, \mathrm{n} \geq 3$ independent measurements. (B) Tubulin polymerization as measured by scattered light at $340 \mathrm{~nm}$ as a function of time. See Table S4 for fit parameters. Data are presented as mean \pm SD following normalization, $\mathrm{n}=3$ independent measurements. Arrows indicate depolymerization at 4 ${ }^{\circ} \mathrm{C}$.

Fig. 5. Model for regulation of tau:tubulin interactions. The PRR (orange) binds tubulin tightly and stoichiometrically, negatively regulated by the NTD (blue). The MTBR-R' (yellow) increases the local tubulin concentration through distributed, weak interactions, enhancing the polymerization capacity of tau. The C-terminus is colored black. Increasing both tau and tubulin concentrations favor polymerization. 


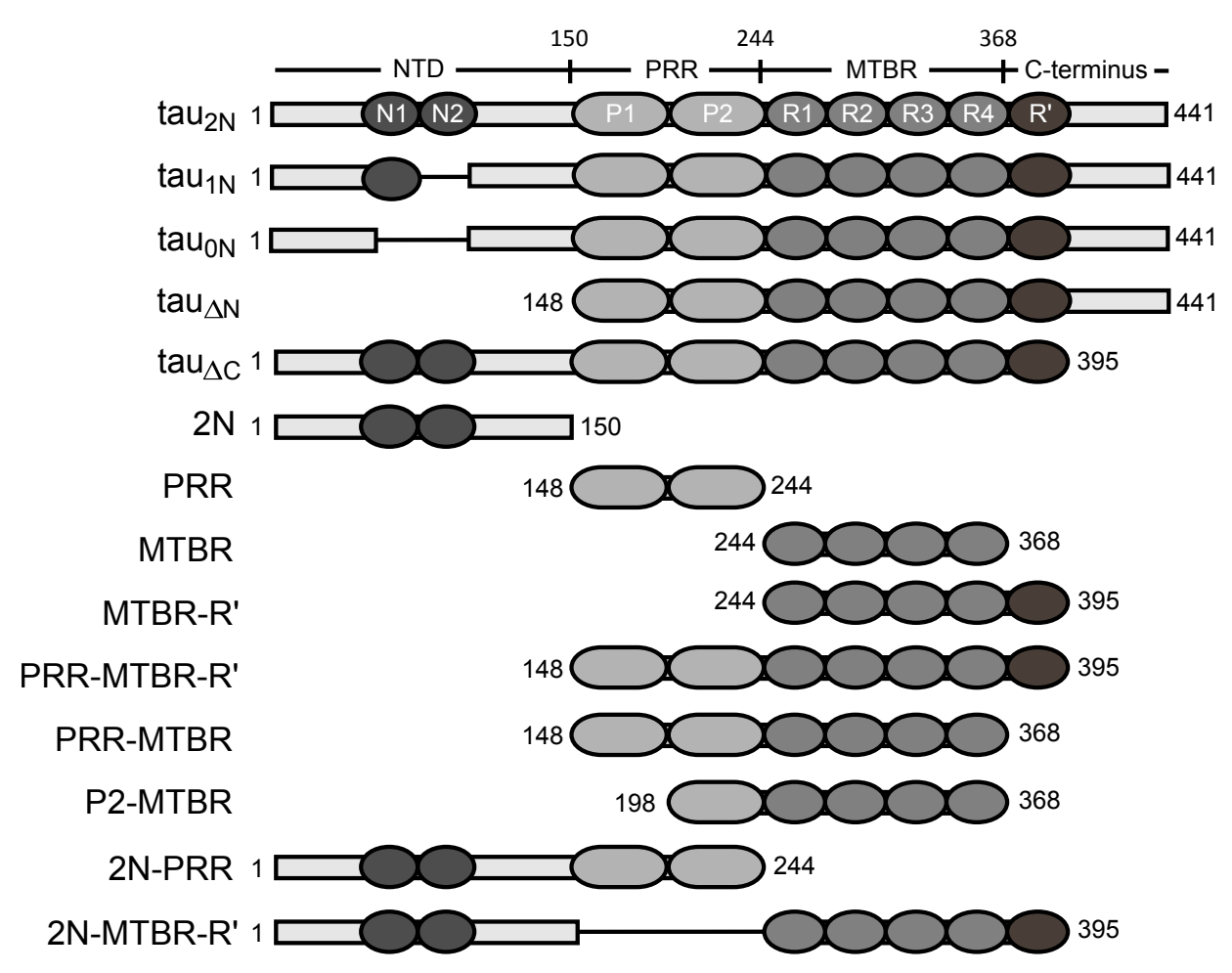

Fig. 1: Schematics of tau constructs. 
A

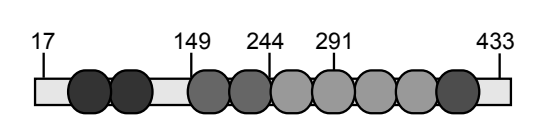

C

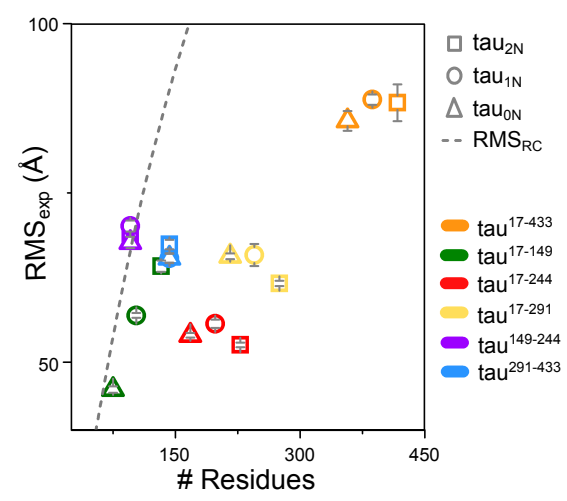

B
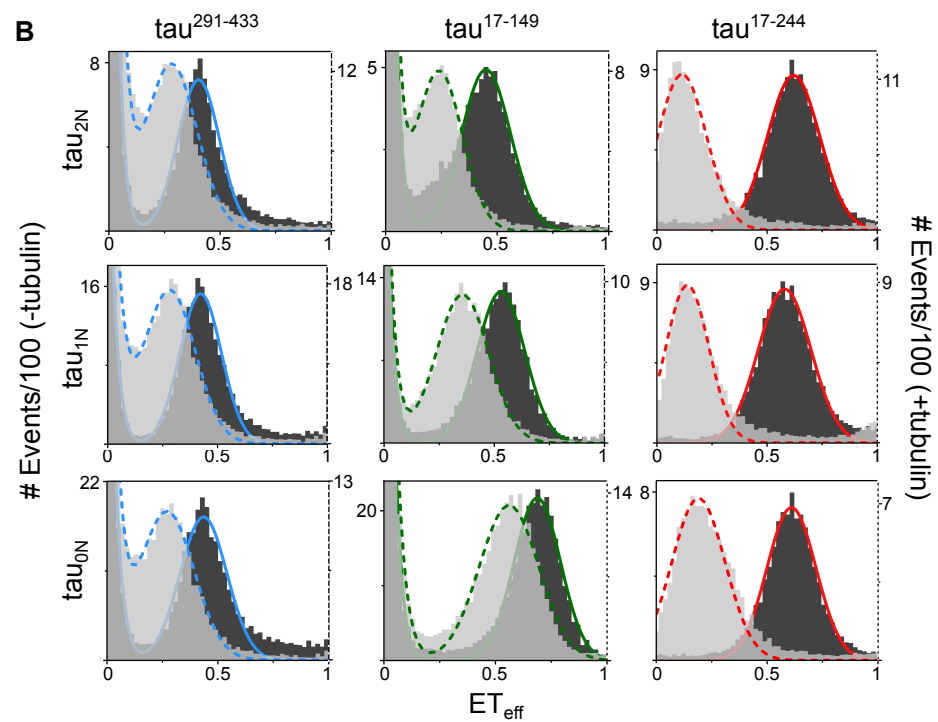

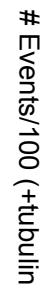

Fig. 2. SmFRET of tau N-terminal isoforms. 

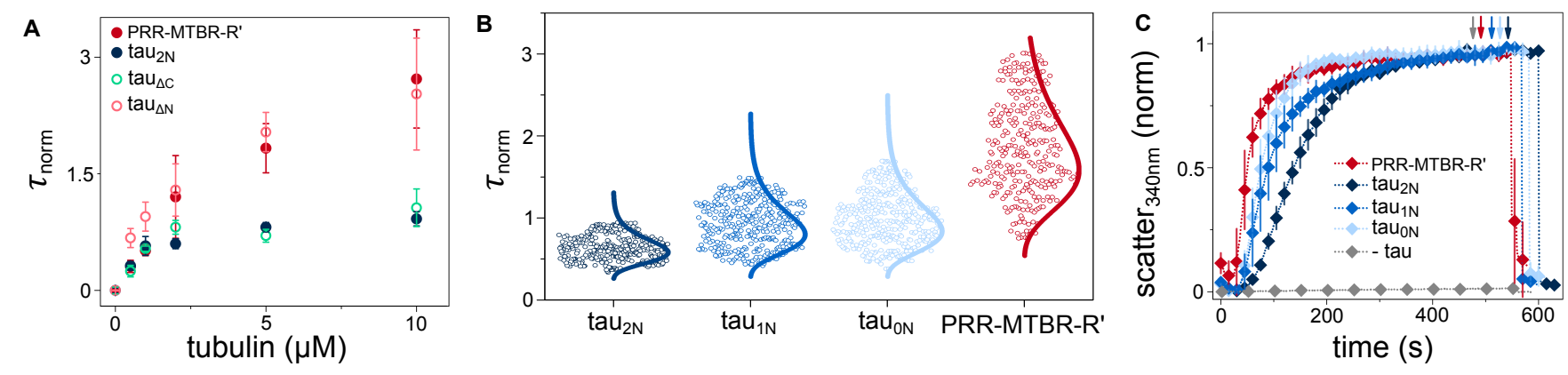

Fig. 3. Inhibition of binding and polymerization by the NTD. 

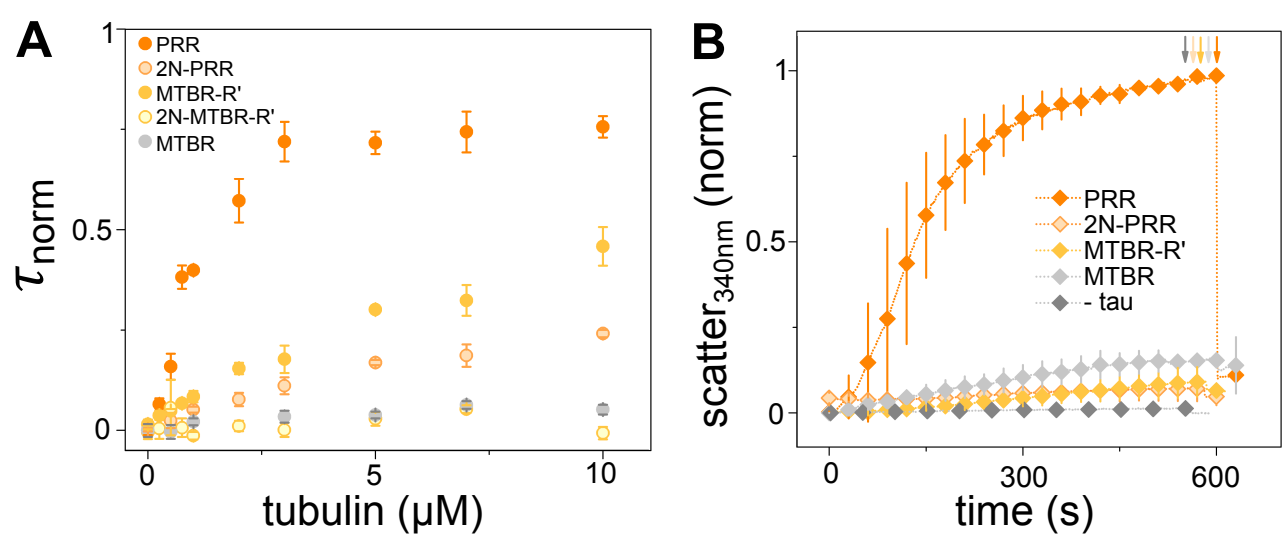

Fig. 4. Independent polymerization capacity of the PRR, regulated by the NTD. 


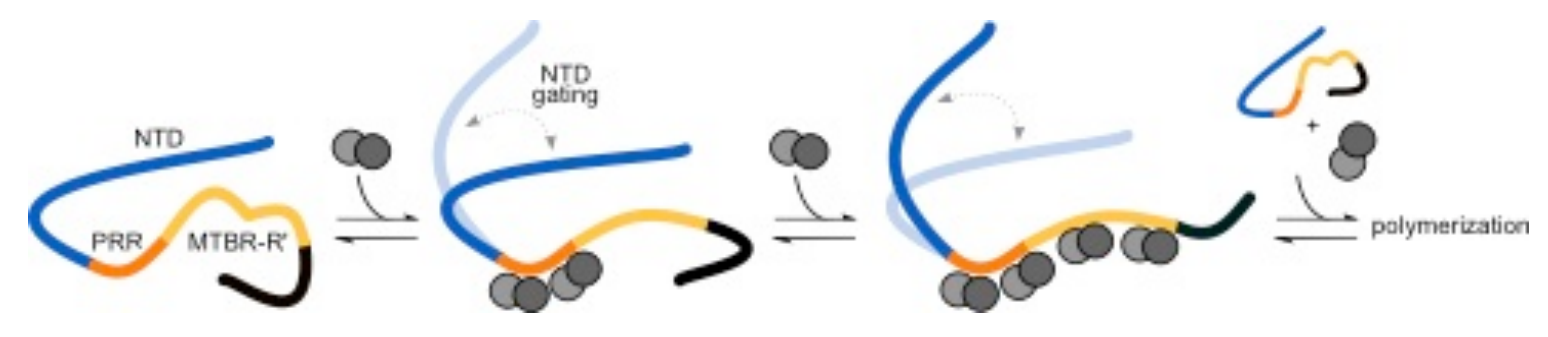

Fig. 5. Model for regulation of tau:tubulin interactions 


\section{SI Appendix}

\section{Tubulin purification and handling}

Tubulin was purified from fresh bovine brains as described in (1). Purified tubulin was snap-frozen in BRB80 (80mM PIPES pH 6.8, $1 \mathrm{mM} \mathrm{MgCl}_{2}, 1 \mathrm{mM}$ EGTA). Prior to use, frozen aliquots were rapidly thawed and then clarified at 100,000xg for 6 minutes. BioSpin 6 columns (BioRad) were used to buffer exchange tubulin into phosphate buffer. The tubulin absorbance at $280 \mathrm{~nm}$ was converted to concentration using a molar extinction coefficient of $115,000 \mathrm{M}^{-1} \mathrm{~cm}^{-1}$. Tubulin was used within 2 hours following clarification.

\section{Tau cloning, purification, and labeling}

The parent tau plasmid encodes for longest tau isoform, tau $2 \mathrm{~N}$. It includes an N-terminal His-tag with a tobacco etch virus (TEV) protease cleavage site for purification (2). The native cysteines, C291 and C322, are mutated to serine to allow for the introduction of cysteines at desired location for site-specific labeling. $\mathrm{Tau}_{1 \mathrm{~N}}$ and tau $\mathrm{u}_{\mathrm{N}}$ were generated using deletion cloning from the tau ${ }_{2 \mathrm{~N}}$ plasmid. The nicked DNA fragments were fused using T4 DNA ligase (New England Biolabs) and T4 Polynucleotide kinase (New England Biolabs). The remaining tau fragments were generated using either site-directed mutagenesis to introduce stop codons and cysteines, deletion cloning of the remaining tau amino acids within the parent tau vector or a combination of the two techniques.

For all constructs longer than 200 residues, tau protein expression was induced with 1mM IPTG at OD $\sim 0.6$ overnight at $16^{\circ} \mathrm{C}$. For constructs $<200$ residues, tau protein expression was induced with $1 \mathrm{mM}$ IPTG at OD $\sim 0.8$ for $4-5$ hours at $37^{\circ} \mathrm{C}$. Purification was based on previously reported methods (2). Briefly, cells were lysed by sonication, and the cell debris pelleted by centrifugation. The supernatant was incubated with Ni-NTA resin (Qiagen or BioRad) and the recombination protein was bump eluted with $500 \mathrm{mM}$ imidazole. The His-tag was removed by incubation lab purified TEV proteinase for either 4 hours at $20{ }^{\circ} \mathrm{C}$ (constructs $<200$ residues) or overnight at $4{ }^{\circ} \mathrm{C}$ (constructs $>200$ residues). Uncleaved protein was removed by a second pass over the Ni-NTA column. Remaining contaminants were removed using size exclusion chromatography on a HiLoad 16/600 Superdex 200 Column (GE LifeSciences). Proteins that did not require fluorescent labeling were buffer exchanged using Amicon concentrators (Millipore) into the final assay buffer of interest, aliquoted and snap frozen for storage at $-80{ }^{\circ} \mathrm{C}$. Due to the small size and lack of aromatic residues, P1 and P2 were TEV-cleaved as described above but after the fluorescent labeling (below). All other proteins were labeled following elution from the size exclusion column.

Site specific labeling of tau for FRET or FCS measurements was carried out as described previously (2). Briefly, tau was incubated with $1 \mathrm{mM}$ DTT for 30 minutes, and then buffer exchanged into labeling 
buffer $(20 \mathrm{mM}$ Tris $\mathrm{pH}$ 7.4, $50 \mathrm{mM} \mathrm{NaCl}$, and $6 \mathrm{M}$ guanidine $\mathrm{HCl})$. For FRET labeled constructs, the donor fluorophore, Alexa Fluor 488 maleimide (Invitrogen), was added at sub-stoichiometric ratios (0.3-0.5x), and incubated at room temperature for 15 minutes. A 3-fold molar excess of the acceptor fluorophore, Alexa Fluor 594 maleimide (Invitrogen) was added and the reaction was incubated for another 10 minutes at room temperature, and then moved to $4{ }^{\circ} \mathrm{C}$ for overnight incubation. For FCS labeled constructs, Alexa Fluor 488 maleimide was added in 3-fold molar excess and incubated at room temperature for 10 minutes, followed by overnight incubation at $4{ }^{\circ} \mathrm{C}$. Labeling reactions were protected from ambient light and with constant stirring; the dye was added dropwise. The labeled protein was buffer exchanged into $20 \mathrm{mM}$ Tris $\mathrm{pH} 7.4$ and $50 \mathrm{mM} \mathrm{NaCl}$ and unreacted dye was removed using HiTrap Desalting Columns (GE Life Sciences). Labeled protein was aliquoted and snap frozen for storage at $-80^{\circ} \mathrm{C}$.

\section{FCS instrument and data analysis}

All FCS measurements were preformed on our home built instrument, as described previously (3). Prior to entering the inverted Olympus 1X-71 Microscope (Olympus), the laser power was adjusted to $\sim 5$ $\mu \mathrm{W}$ (488 nm diode-pumped solid-state laser, Spectra-Physics) and focused into the sample via a 60x water objective (Olympus). Fluorescence emission from the sample was collected through the objective, separated from excitation light by a Z488RDC long pass dichroic and a $500 \mathrm{~nm}$ long pass filter (Chroma). The filtered emission was focused the aperture of a $50 \mu \mathrm{m}$ diameter optical fiber (OzOptics) coupled to an avalanche photodiode (Perkin-Elmer). A digital correlator (FLEX03LQ-12, Correlator.com) generated the autocorrelation curves.

Measurements were made in 8-chamber Nunc coverslips (Thermo-Fisher) passivated by incubation with (ethylene glycol)poly(L-lysine) (PEG-PLL)(4). The labeled tau (15-25 nM) and tubulin (concentrations vary) were incubated in chambers for 5 minutes prior to measurement. Unless otherwise noted, all FCS experiments were carried out in phosphate buffer $(20 \mathrm{mM}$ phosphate $\mathrm{pH} 7.4,20 \mathrm{mM} \mathrm{KCl}, 1 \mathrm{mM} \mathrm{MgCl}, 0.5$ mM EGTA, $1 \mathrm{mM}$ DTT) at $20{ }^{\circ} \mathrm{C}$. Multiple (20-40) 10 second autocorrelation curves were collected per sample and fit to a single-component 3D diffusion equation (Eq. S1):

$$
G(\tau)=\frac{1}{N\left(1+\frac{\tau}{\tau_{D}}\right)} \sqrt{\frac{1}{1+\frac{s^{2} \tau}{\tau_{D}}}}
$$

where $G(\tau)$ is the autocorrelation function as a function of time $(\tau), \tau_{D}$ is the translational diffusion time of the labeled molecules and $N$ is the average number of fluorescent species. For our instrument, the ratio of the radial to axial dimensions of the focal volume (s) was determined to be 0.2 and consequently fixed for analysis. The recorded intensity trace is divided by $\mathrm{N}$ to give counts per molecule (CPM) in $\mathrm{kHz}$. 
For some tau constructs, high tubulin concentrations $(>2 \mu \mathrm{M})$ result in the formation of large, bright species (Figs. 3, S2 and S4). These species are not present in the traces of protein in the absence of tubulin (Figs. S8 and S9A). A prior study from our lab demonstrated these species are tau:tubulin specific, electrostatically sensitive and reversible (3). For P2-MTBR, increasing the $\mathrm{KCl}$ concentration in our phosphate buffer to $300 \mathrm{mM}$ - previously seen by NMR to disrupt interactions between the PRR and tubulin - results in disassembly of the larger species (Table S5) (5). In the case of PRR-MTBR-R', these species persist even at $300 \mathrm{mM} \mathrm{KCl}$ suggesting the binding is either tighter or has a more hydrophobic character (Table S5).

The individual autocorrelation curves arising from these larger assemblies disproportionally weight the averaged autocorrelation curves used in the analysis described above (Table S3 and Fig. S9). Working under the premise that removal of these outliers would allow for a more meaningful analysis of the majority tau:tubulin complexes, we developed an algorithm to remove aberrant curves, broadly following the approach we described previously (3). Individual autocorrelation curves were fit with Eq. S1 and assessed the goodness of fit using least-squares $X^{2}=\left[\mathrm{G}(\tau)_{\text {fit }}-\mathrm{G}(\tau)_{\text {raw }}\right]^{2}$ with a tolerance of $X^{2}=0.0001$ for a consecutive run of $75 \mathrm{~ms}$. In other words, if the fit deviated beyond the $X^{2}$ for more than $75 \mathrm{~ms}$, the curve was discarded. This process removes $99.5 \%$ of curves that cannot be accurately fit using Eq. S1. The frequency of these aberrant curves is $\sim 3 \%$.

Autocorrelation curves arising from larger assemblies that pass this initial criterion still skew the data towards slower diffusion times (Table S3). Descriptive statistics of these diffusion times are reported in Table S3 as "pre-filtering”. In some cases, such as PRR-MTBR-R' the measured $\tau_{\mathrm{D}}$ could be as large as $\sim 14$ ms and up to $4 x$ brighter than unbound tau (Fig. S9B,C). Although of potential interest in another context, these species do not represent the majority of the tau:tubulin complexes of interest here. These outliers were removed in an iterative fashion by testing the individual curves using an Anderson-Darling statistical test for either a lognormal or normal distribution. Diffusion times above or below the interquartile range were removed until a stable population was reached and no more curves were removed from the dataset. We did not: (1) enforce a lognormal or normal distribution on the data-set prior to outlier removal; nor (2) continue or use outlier removal if the population is normal or lognormal after testing. This iterative function is demonstrated for tau $\mathrm{u}_{\mathrm{N}}$ in the absence (Fig. S9D) or presence of $10 \mu \mathrm{M}$ tubulin (Fig. S9E). The initial iteration simply tests for normality (seen by the straight line in Fig. S9D for a single iteration). This results the removal approximately $\sim 15-20 \%$ of curves that passed the initial goodness-of-fit filtering from the data set (Table S3).

The fit parameters from the individual filtered curves are presented in Figs. $3 B\left(\tau_{\text {norm }}\right)$ and S2B (CPM), and the descriptive statistics of these values are listed in Table S3. There is a general correlation 
showing that tau:tubulin complexes with larger $\tau_{\mathrm{D}} \mathrm{S}$ also had larger CPMs, reflecting the presence of multiple tau molecules in these assemblies (Fig. S2C). In order to allow for straightforward comparison between tau isoforms, we also averaged the filtered curves from each independent measurement and fit the average curve with Eq. S1. These $\tau_{\mathrm{D}}$ values obtained from these fits are reported in Table S5 for saturating points, and are graphed in figures with FCS binding curves (Figs. 3, 4, S2, S3, S5, S6 and S7).

Tau constructs of different lengths have different diffusion times. To allow for straightforward comparison of the extent of binding between the various constructs, the diffusion times for each construct in the presence of tubulin ( $\tau_{D}^{\text {bound }}$ ) were normalized to that of the construct in the absence of tubulin $\left(\tau_{D}^{\text {free }}\right)$ as follows:

$$
\tau_{\text {norm }}=\frac{\tau_{D}^{\text {bound }}-\tau_{D}^{\text {free }}}{\tau_{D}^{\text {free }}}
$$

Both $\tau_{D}$ and $\tau_{\text {norm }}$ are listed in Table S5.

In contrast to other constructs, binding of the PRR to tubulin has a sigmoidal shape with a saturation plateau beyond $2 \mu \mathrm{M}$ tubulin (Fig. S3). The binding curve fits to the Hill equation:

$$
\tau_{\text {norm }}=\tau_{\text {norm }}^{\max }+\tau_{\text {norm }}^{\max } \frac{[t u b]^{n}}{K_{D}+[t u b]^{n}}
$$

where $\tau_{\text {norm }}^{\max }$ is the normalized diffusion time for tau:tubulin measured at $10 \mu \mathrm{M}$ tubulin, $n$ is the Hill coefficient and reflects the extent of cooperativity, $K_{D}$ is the apparent dissociation constant and [tub] is the concentration of tubulin. Fitting our data with this equation yields, $n=1.7 \pm 0.2$ and $K_{D}=900 \mathrm{nM} \pm 10 \mathrm{nM}$. In our previous work, we used the engineered protein construct RB3, which binds tubulin with 1:2 RB3: tubulin dimer stoichiometry to determine the expected $\tau_{D}$ of a 1:2 protein:tubulin complex $(3,6)$. Here, the $\tau_{\mathrm{D}}$ measured for the PRR at $10 \mu \mathrm{M}$ tubulin $(0.82 \pm 0.03 \mathrm{~ms})$ is consistent with a 1:2 tau:tubulin dimer stoichiometry. This observation, coupled with the cooperativity seen in Hill equation fit, strongly supports the presents of two tubulin-dimer binding sites in the PRR.

\section{FRET instrument and analysis}

For FRET histograms where the protein signal was readily distinguishable from the 'zero-peak' (7), arising from imperfect labeling were carried out on our lab built instrument, as described above with a few modifications. The laser power is adjusted to $\sim 30 \mu \mathrm{W}(488 \mathrm{~nm}$ diode-pumped solid-state laser, SpectraPhysics) prior to entering the microscope. Donor and acceptor photons were separated using a HQ585 long pass dichroic and further selected with ET525/50M band pass and HQ600 long pass filters (Chroma). For each path, the emission was focused onto the aperture of a $100 \mu \mathrm{m}$ diameter optical fiber (OzOptics) coupled to an avalanche photodiode (Perkin-Elmer). Time traces were collected in $1 \mathrm{~ms}$ time bins for 1 
hour. As described above, measurements were carried out in PEG-PLL coated Nunc chambers with 20-40 pM labeled tau following 5 minutes incubation with tubulin.

To differentiate photon bursts arising from transit of a labeled molecule from background fluorescence, a photon count threshold of 30 counts/ms was applied. For each burst, the energy transfer efficiency $\left(\mathrm{ET}_{\text {eff }}\right)$ calculated using a lab-based written software (MATLAB) according to the following equation $(8,9)$ :

$$
E T_{e f f}=\frac{\left(I_{a}-\beta I_{d}\right)}{\left(I_{a}-\beta I_{d}\right)+\gamma\left(I_{d}+\beta I_{d}\right)}
$$

where $I_{a}$ is the intensity of the acceptor photons and $I_{d}$ is the intensity of the donor photons. Within our system, the bleed through of the donor channel into the acceptor channel $(\beta)$ and the difference in the total quantum efficiency of the system and fluorophores $(\gamma)$ were determined using Alexa Fluor 488 hydrazine (Invitrogen) and Alexa Fluor 594 hydrazine (Invitrogen) and fixed for analysis. Due to variation in instrument build and detector efficiency over the course of the study, $\beta$ and $\gamma$ were regularly re-determined and checked with DNA standards of 10, 14, and 18 bases labeled with Alexa Fluor 488 and Alexa Fluor 594 (Integrated DNA Technologies). The energy efficiencies were then binned, and the histograms fit using a sum of Gaussians in Origin. One Gaussian described the "zero-peak" (donor-only fluorescence) and the second peak described donor and acceptor labeled protein (main peak fit listed in Table S2). In some cases, the distribution was asymmetric ( $\operatorname{such}$ as tau ${ }^{17-149}$ ). In these cases, three Gaussians were used to fit the data. The Gaussian that fit the dominant peak is reported in Table S2.

At some of the labeling positions, the proteins gave rise to low energy efficiencies with overlap with zero-peak, making it difficult to accurately determine the peak $E T_{\text {eff }}$ for the protein sample. To separate donor-only labeled species from the low energy, donor and acceptor labeled species, measurements were repeated on a commercial MicroTime 200 time-resolved confocal microscope (Picoquant) using its pulsed interleaved excitation FRET (PIE-FRET) mode. The power of the excitation lasers (485 nm and $562 \mathrm{~nm}$ ) were matched $\sim 30 \mu \mathrm{W}$ at $40 \mathrm{MHz}$. The fluorescence emission was focused through a $100 \mu \mathrm{m}$ pinhole and collected by avalanche photodiode. Fluorescence emission of the donor and acceptor fluorophores were separated using a HQ585 long pass dichroic and further selected with ET525/50M band pass and HQ600 long pass filters. SymphoTime 64 software was used to analyze the photon bursts to yield both the $E T_{\text {eff }}$ and stoichiometry factors for each burst, using photon threshold, binning and experimentally determined $\beta$ and $\gamma$ values as described previously. The binned histograms were fit as described above. 


\section{Tubulin polymerization Assay}

Polymerization of soluble tubulin was measured by monitoring the increase in scattered light at 340 $\mathrm{nm}$. The tubulin was clarified as described above and buffer exchanged in phosphate buffer at $\mathrm{pH} 6.9$ immediately prior to use. For polymerization reactions, $10 \mu \mathrm{M}$ tubulin was incubated with $5 \mu \mathrm{M}$ tau (for $\operatorname{tau}_{2 \mathrm{~N}}, \operatorname{tau}_{1 \mathrm{~N}}, \operatorname{tau}_{0 \mathrm{~N}}$, and PRR-MTBR-R') or $10 \mu \mathrm{M}$ tau (for PRR, 2N-PRR, MTBR, and MTBR-R') for 5 minutes on ice prior to the addition of $1 \mathrm{mM}$ GTP. Immediately after the addition of GTP, the reaction was transferred to a warmed cuvette and the reaction was monitored for 10 minutes at $37{ }^{\circ} \mathrm{C}$ in a fluorometer (Fluorolog FL-1039/40, Horiba) with a photon counting module (SPEX DM302, Horiba) with both excitation and emission wavelengths set to $340 \mathrm{~nm}$. Following polymerization, the samples were quickly returned to $4{ }^{\circ} \mathrm{C}$; cold depolymerization is evidence that the proteins are not aggregated.

The curves were normalized to the brightest intensity within a given curve, and fit in Origin with:

$$
y=\frac{1}{1+e^{\frac{t-t_{1 / 2}}{d t}}}
$$

where $\mathrm{y}$ is the normalized fluorescence intensity, $\mathrm{t}$ is time, $t_{1 / 2}$ is the polymerization half-time and $d t$ is the time constant. The mean and standard deviation of the $t_{1 / 2}$ values are listed in Table S4. The plotted graphs represent the average of the normalized triplicate with standard deviation. 
A

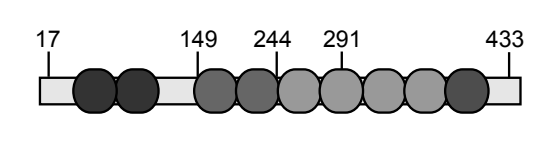

c

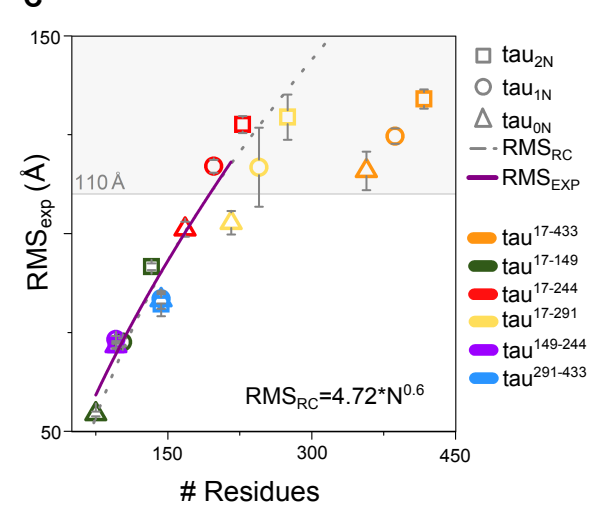

B

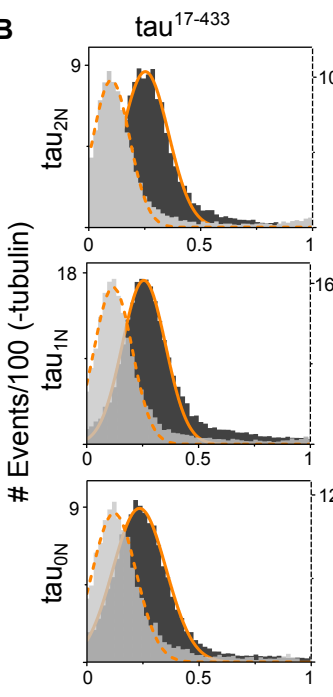

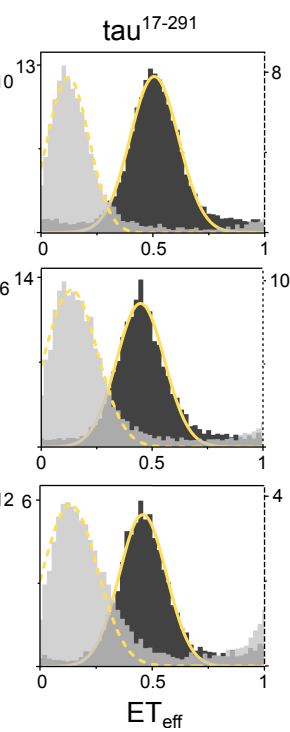

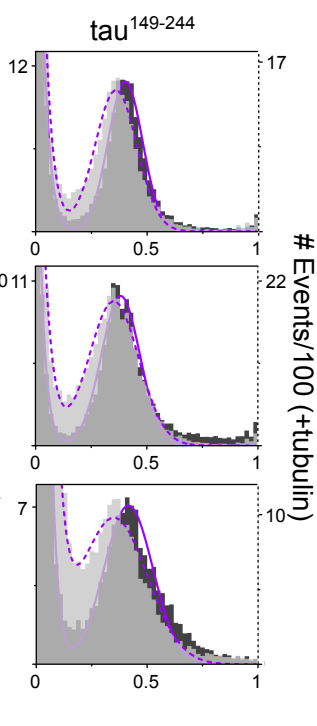

Fig. S1. SmFRET of tau N-terminal isoforms. (A) Schematic of reference construct tau $2 \mathrm{~N}$ with residues used for labeling for smFRET measurements are indicated. (B) SmFRET histograms of tau $2 \mathrm{~N}$, tau $\mathrm{u}_{1 \mathrm{~N}}$ and tau $0 \mathrm{~N}$ labeled at residues 17 and 433 (left), 17 and 291 (center), 149 and 244 (right) in the absence (dark: left axis) and presence (light: right axis) of $10 \mu \mathrm{M}$ tubulin. Histograms for tau labeled at residues 291 and 433,17 and 244, 149 and 244 are in Fig. 2. Fits to the data as a sum of Gaussian distributions as described in the SI Appendix are shown. (C) For measurements in the presence of tubulin, the root-mean-square distances (RMS) between labeling positions calculated from a Gaussian chain model ( $\mathrm{RMS}_{\text {exp }}$ ) are plotted (10). Data are presented as mean $\pm \mathrm{SD}, \mathrm{n} \geq 3$ independent measurements. For histograms with mean $\mathrm{ET}_{\text {eff }}$ values $<0.16$ (shaded; corresponding to $\mathrm{RMS}_{\exp } \geq 110 \AA$ ), it is not possible to accurately calculate $\mathrm{RMS}_{\exp }$ because the average distance between the fluorophores is to large for the dye pair used. For $\mathrm{RMS}_{\exp }$ values $<110 \AA$, the data was fit using an exponential curve $\left(\mathrm{RMS}_{\mathrm{exp}}\right)$. For reference, the RMS calculated for a random coil $\left(\mathrm{RMS}_{\mathrm{RC}}\right)$ as in Reference (11) is indicated by the gray dashed line. See Table S2 for, numerical values of $\mathrm{ET}_{\mathrm{eff}}$ mean $\pm \mathrm{SD}, \mathrm{RMS}_{\mathrm{exp}} \pm \mathrm{SD}$ and $\mathrm{RMS}_{\mathrm{RC}}$ for each construct. 

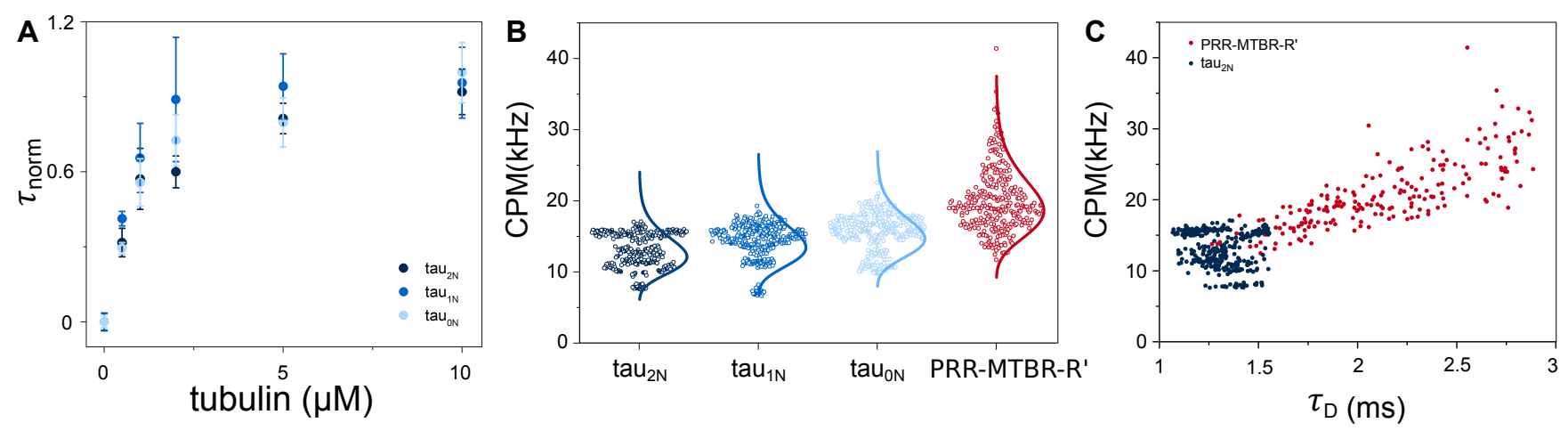

Fig. S2. Relative binding and brightness of tau N-terminal isoforms. (A) The average $\tau_{\text {norm }}$ of tau constructs $\operatorname{tau}_{2 \mathrm{~N}}$ (replotted from Fig. 2A for comparison), $\operatorname{tau}_{1 \mathrm{~N}}$ and $\operatorname{tau}_{0 \mathrm{~N}}$ are plotted against increasing tubulin concentration. Data are presented as mean $\pm \mathrm{SD}, \mathrm{n} \geq 3$ independent measurements. See SI Appendix for details of data analysis. See Table S5 for numerical values for $\tau_{\mathrm{D}}$ and $\tau_{\text {norm }}$ at $10 \mu \mathrm{M}$ tubulin. (B) The corresponding CPM of individual autocorrelation curve fits of tau $2 \mathrm{~N}, \operatorname{tau}_{1 \mathrm{~N}}$, tau $\tan _{0 \mathrm{~N}}$ and PRR-MTBR-R' incubated with $10 \mu \mathrm{M}$ tubulin from Fig. 3B with lognormal distribution overlaid. (C) Scatterplot of CPM versus $\tau_{\mathrm{D}}$ of PRR-MTBR-R' and tau $2 \mathrm{~N}$ from panels $(B)$ and Fig. $3 \mathrm{~B}$, respectively. 


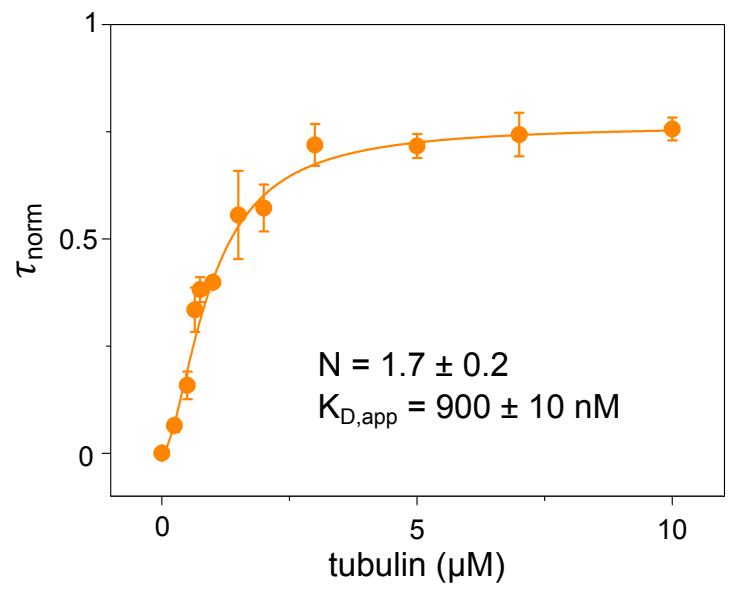

Fig. S3. Cooperative binding by PRR. The average $\tau_{\text {norm }}$ and standard deviation of the PRR is plotted against increasing tubulin concentration (re-plotted from Fig. 4A with additional tubulin concentrations). The binding curve was fit to the Hill equation (Eq. S3) was to yield $n=1.7 \pm 0.2$ and the apparent $K_{D}=900 \pm$ $10 \mathrm{nM}$. Data are presented as mean $\pm \mathrm{SD}, \mathrm{n} \geq 3$ independent measurements. See SI Appendix for details of data analysis. See Table S5 for numerical values for $\tau_{\mathrm{D}}$ and $\tau_{\text {norm }}$ at $10 \mu \mathrm{M}$ tubulin. 


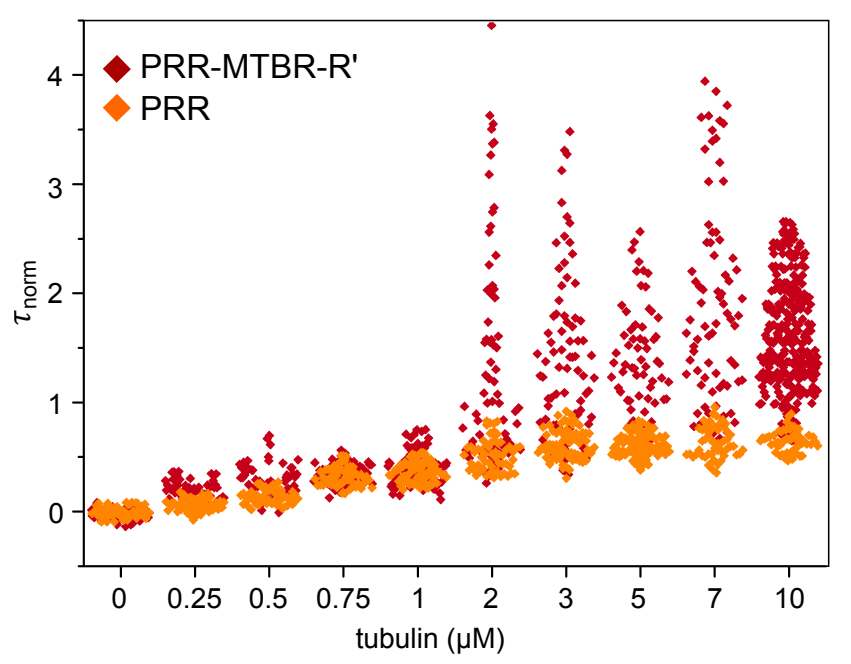

Fig. S4. Comparison of heterogeneity of PRR and PRR-MTBR-R'. The $\tau_{D}$ values from fitting individual autocorrelation curves for PRR-MTBR-R' (from Fig. 3) and PRR (from Fig. 4) is plotted as a scatterplot against increasing tubulin concentration. 


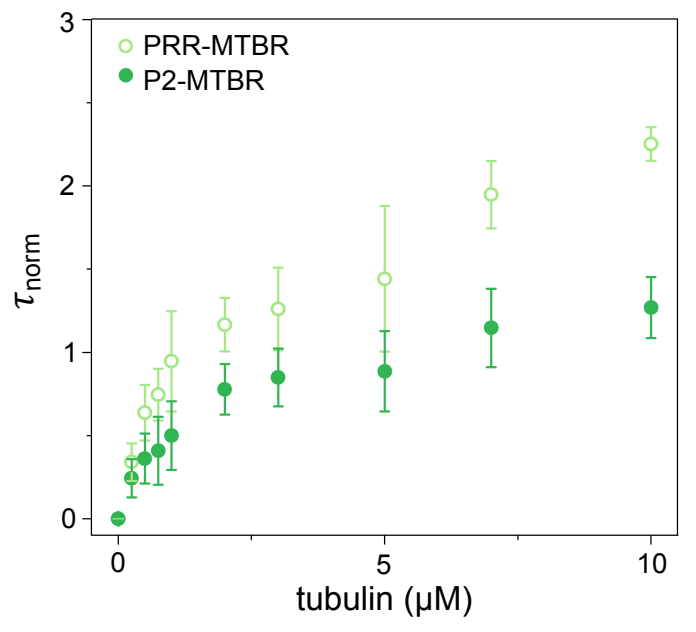

Fig. S5. Impact of P1 on binding of the PRR-MTBR. The $\tau_{\text {norm }}$ of tau constructs PRR-MTBR and P2MTBR measured by FCS are plotted against increasing tubulin concentration. Data are presented as mean \pm $\mathrm{SD}, \mathrm{n} \geq 3$ independent measurements. See SI Appendix for details of data analysis. See Table S5 for numerical values for $\tau_{\mathrm{D}}$ and $\tau_{\text {norm }}$ at $10 \mu \mathrm{M}$ tubulin. 


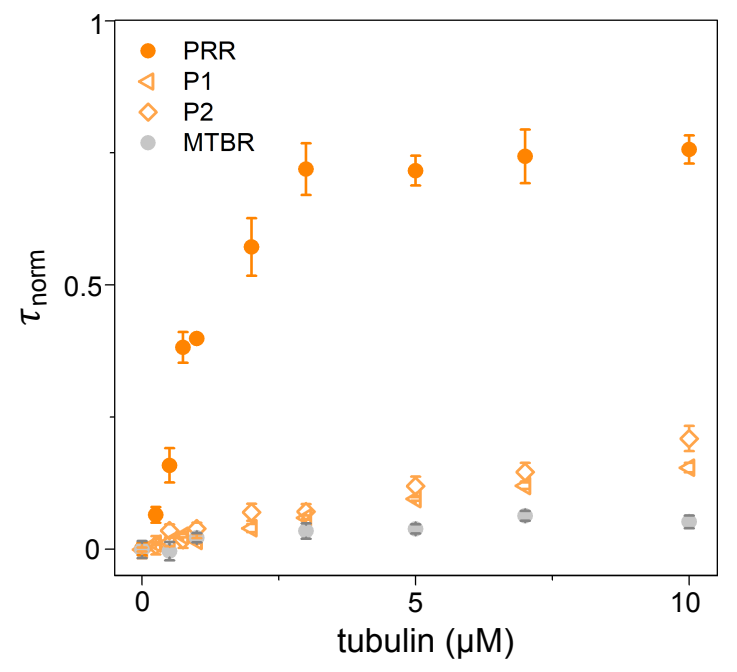

Fig. S6. Relative binding of P1 and P2. The average $\tau_{\text {norm }}$ and standard deviation of FCS tau constructs P1 and $\mathrm{P} 2$ are plotted against increasing tubulin concentration. Data are presented as mean $\pm \mathrm{SD}, \mathrm{n} \geq 3$ independent measurements. Binding curves of the PRR and MTBR (both from Fig. 4A) are plotted for comparison. See SI Appendix for details of data analysis. See Table S5 for numerical values for $\tau_{\mathrm{D}}$ and $\tau_{\mathrm{norm}}$ at $10 \mu \mathrm{M}$ tubulin. 


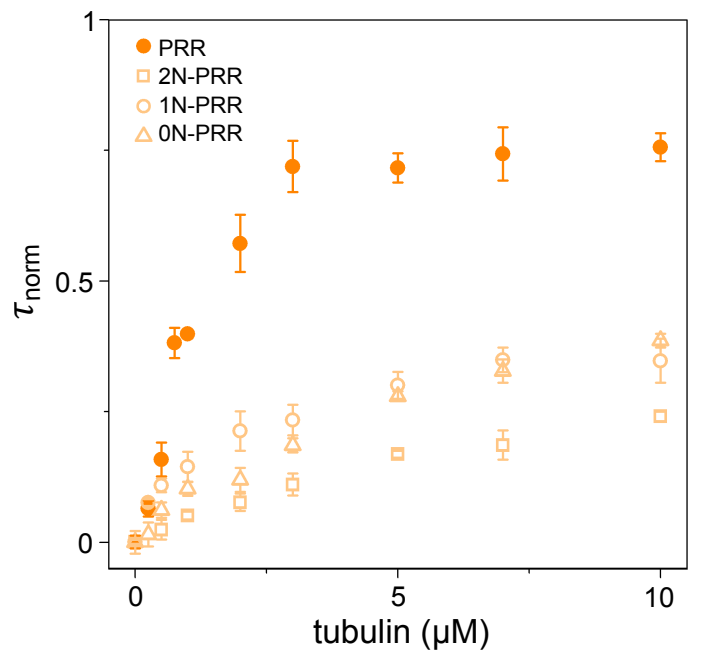

Fig. S7. Impact of N-terminal inserts on the binding of PRR. The $\tau_{\text {norm }}$ of tau constructs $1 \mathrm{~N}-\mathrm{PRR}$ and $0 \mathrm{~N}-$ PRR are plotted against increasing tubulin concentration. Tau constructs PRR and 2N-PRR are re-plotted from Fig. 4A for comparison. Data are presented as mean $\pm \mathrm{SD}, \mathrm{n} \geq 3$ independent measurements. See $S I$ Appendix for details of data analysis. See Table S5 for numerical values for $\tau_{\mathrm{D}}$ and $\tau_{\text {norm }}$ at $10 \mu \mathrm{M}$ tubulin. 

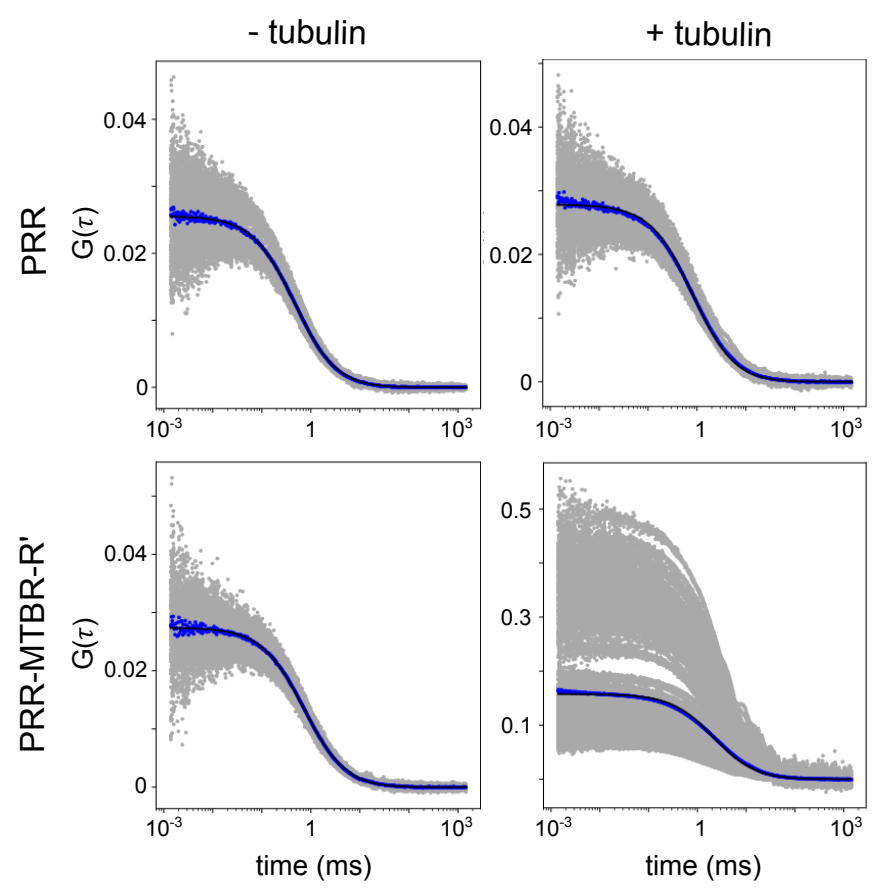

Fig. S8. Heterogeneity in tubulin-bound PRR-MTBR-R'. Individual autocorrelation curves (gray dots) are plotted for PRR (upper) and PRR-MTBR-R' (lower) in the absence (left) or presence (right) of $10 \mu \mathrm{M}$ tubulin. Averaged curves are shown with blue dots and fits of the averaged curves to Eq. S1 are in black. 

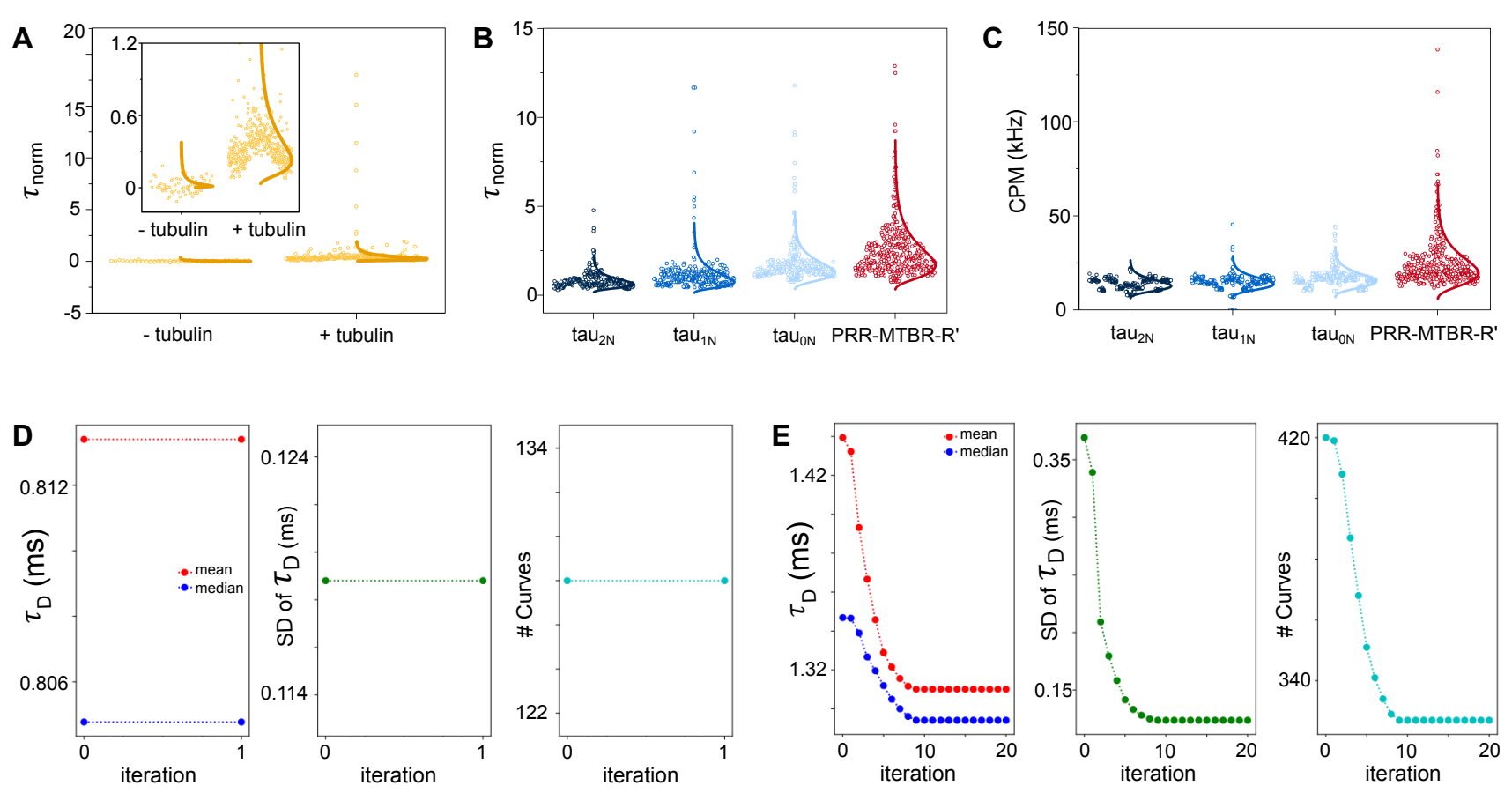

Fig. S9. Filtering algorithm. (A) $\tau_{\text {norm }}$ of MTBR-R' in the absence or presence of tubulin without any filtering. Inset is a magnification of the y-axis for low $\tau_{\text {norm }}$ (B) $\tau_{\text {norm }}$ of tau $\tan _{2 \mathrm{~N}}, \operatorname{tau}_{1 \mathrm{~N}}$, tau $\mathrm{tan}_{0 \mathrm{~N}}$ and PRR-MTBR$\mathrm{R}^{\prime}$ with $10 \mu \mathrm{M}$ tubulin Fig. 4A after goodness-of-fit filtering, but without iterative filtering (as described in the SI Appendix) with lognormal distribution overlaid. $(C)$ The corresponding CPM (kHz) for each point in (B) (D) Mean and median $\tau_{\mathrm{D}}$ (right), the standard deviation of $\tau_{\mathrm{D}}$ (middle) and number of curves (left) for $\operatorname{tau}_{2 \mathrm{~N}}$ over the iteration number (1) for $\operatorname{tau}_{2 \mathrm{~N}}$ in the absence of tubulin. No curves are selected for elimination in this tau-only sample. (E) Mean and median $\tau_{\mathrm{D}}$ (right), the standard deviation of $\tau_{\mathrm{D}}$ (middle) and number of curves (left) for tau $\tan _{2 \mathrm{~N}}$ in the presence of $10 \mu \mathrm{M}$ tubulin over the iteration number demonstrating convergence of the algorithm (at iteration 9) described in the SI Appendix. 
Table S1. Tau constructs and their corresponding residues.

\begin{tabular}{|c|c|c|}
\hline construct & residues & $\mathbf{C}_{\mathrm{FCS}}$ \\
\hline $\operatorname{tau}_{2 \mathrm{~N}}$ & $1-441$ & 433 \\
\hline $\operatorname{tau}_{1 \mathrm{~N}}$ & $1-441_{\triangle 45-75}$ & 433 \\
\hline $\operatorname{tau}_{0 \mathrm{~N}}$ & $1-441_{\Delta 45-102}$ & 433 \\
\hline $\operatorname{tau}_{\Delta \mathrm{N}}$ & $148-441$ & 433 \\
\hline $\operatorname{tau}_{\Delta} \mathrm{c}$ & $1-395$ & 17 \\
\hline $2 \mathrm{~N}$ & $1-150$ & 17 \\
\hline PRR & $148-244$ & 149 \\
\hline MTBR & $244-372$ & 322 \\
\hline MTBR-R' & 244-395 & 244 \\
\hline PRR-MTBR-R' & $148-395$ & 149 \\
\hline PRR-MTBR & $148-372$ & 149 \\
\hline 2N-PRR & $1-244$ & 17 \\
\hline 1N-PRR & $1-244_{\Delta 45-75}$ & 17 \\
\hline ON-PRR & $1-244_{\Delta 45-102}$ & 17 \\
\hline 2N-MTBR-R' & $1-395_{\Delta 151-253}$ & 17 \\
\hline $\mathrm{P} 1$ & 148-197 & 149 \\
\hline P2 & $198-244$ & 244 \\
\hline P2-MTBR & $198-372$ & 322 \\
\hline
\end{tabular}

All numbering throughout the manuscript is based on tau $\mathrm{u}_{2 \mathrm{~N}}$. Unless otherwise noted, all constructs contain $\mathrm{C} 291 \mathrm{~S}$ and $\mathrm{C} 322 \mathrm{~S}$ mutations. $\mathrm{C}_{\mathrm{FCS}}$ is the residue number mutated to cysteine for labeling for FCS measurements. 
Table S2. SmFRET of N-terminal isoforms

\begin{tabular}{|c|c|c|c|c|c|c|c|c|}
\hline \multirow{2}{*}{ isoform } & \multirow{2}{*}{\multicolumn{2}{|c|}{ labels }} & \multirow{2}{*}{ \# residues } & \multicolumn{2}{|c|}{$\mathrm{ET}_{\text {eff }}$} & \multicolumn{2}{|c|}{$\mathbf{R M S}_{\exp }(\mathbf{A})$} & \multirow{2}{*}{$\mathrm{RMS}_{\mathrm{RC}}(\AA)$} \\
\hline & & & & - tubulin & + tubulin & - tubulin & + tubulin & \\
\hline \multirow{6}{*}{$\operatorname{tau}_{2 \mathrm{~N}}$} & & 433 & 417 & $0.27 \pm 0.02$ & $0.10 \pm 0.01$ & $88 \pm 3$ & $134 \pm 2$ & 181 \\
\hline & 17 & 291 & 275 & $0.52 \pm 0.02$ & $0.11 \pm 0.02$ & $62 \pm 1$ & $129 \pm 6$ & 141 \\
\hline & 17 & 244 & 228 & $0.62 \pm 0.01$ & $0.11 \pm 0.01$ & $52 \pm 1$ & $127 \pm 2$ & 126 \\
\hline & 17 & 149 & 133 & $0.48 \pm 0.01$ & $0.25 \pm 0.01$ & $64 \pm 1$ & $92 \pm 1$ & 91 \\
\hline & 149 & 244 & 96 & $0.43 \pm 0.01$ & $0.39 \pm 0.01$ & $68 \pm 1$ & $72 \pm 1$ & 75 \\
\hline & 291 & 433 & 143 & $0.44 \pm 0.01$ & $0.31 \pm 0.02$ & $67 \pm 1$ & $82 \pm 3$ & 95 \\
\hline \multirow{6}{*}{$\operatorname{tau}_{1 \mathrm{~N}}$} & 17 & 433 & 386 & $0.26 \pm 0.01$ & $0.12 \pm 0.01$ & $89 \pm 1$ & $125 \pm 2$ & 172 \\
\hline & 17 & 291 & 245 & $0.45 \pm 0.01$ & $0.14 \pm 0.02$ & $66 \pm 2$ & $117 \pm 10$ & 131 \\
\hline & 17 & 244 & 198 & $0.58 \pm 0.01$ & $0.14 \pm 0.01$ & $56 \pm 1$ & $117 \pm 2$ & 115 \\
\hline & 17 & 149 & 103 & $0.56 \pm 0.01$ & $0.39 \pm 0.01$ & $57 \pm 1$ & $73 \pm 1$ & 78 \\
\hline & 149 & 244 & 96 & $0.41 \pm 0.01$ & $0.38 \pm 0.01$ & $70 \pm 1$ & $73 \pm 1$ & 75 \\
\hline & 291 & 433 & 143 & $0.46 \pm 0.01$ & $0.30 \pm 0.01$ & $65 \pm 1$ & $83 \pm 1$ & 95 \\
\hline \multirow{6}{*}{$\operatorname{tau}_{0 \mathrm{~N}}$} & 17 & 433 & 358 & $0.28 \pm 0.02$ & $0.15 \pm 0.01$ & $86 \pm 1$ & $\overline{1115 \pm 5}$ & 165 \\
\hline & 17 & 291 & 216 & $0.46 \pm 0.01$ & $0.19 \pm 0.01$ & $67 \pm 1$ & $103 \pm 3$ & 122 \\
\hline & 17 & 244 & 168 & $0.61 \pm 0.01$ & $0.20 \pm 0.01$ & $54 \pm 1$ & $101 \pm 2$ & 105 \\
\hline & 17 & 149 & 75 & $0.72 \pm 0.01$ & $0.60 \pm 0.01$ & $46 \pm 1$ & $54 \pm 1$ & 65 \\
\hline & 149 & 244 & 96 & $0.44 \pm 0.01$ & $0.40 \pm 0.01$ & $68 \pm 1$ & $71 \pm 1$ & 75 \\
\hline & 291 & 433 & 143 & $0.46 \pm 0.01$ & $0.30 \pm 0.02$ & $65 \pm 1$ & $83 \pm 2$ & 95 \\
\hline
\end{tabular}

Peak $\mathrm{ET}_{\text {eff }}$ values from fits to histograms as shown in Fig. 2 and Fig. S1 from measurements in the absence and present of $10 \mu \mathrm{M}$ tubulin. $\mathrm{RMS}_{\text {exp }}$ calculated from mean $\mathrm{ET}_{\text {eff }}$ values as described in the SI Appendix and $\mathrm{RMS}_{\mathrm{RC}}$ calculated from at theoretical random coil model for the number of residues noted (10). Values are mean $\pm \mathrm{SD}$ for $\geq 3$ independent measurements. Labels identifies the residues mutated to cysteines for site-specific labeling. Errors are the standard deviation of the mean from at least three independent measurements. 
Table S3. Descriptive statistics of tau:tubulin

\begin{tabular}{c|ccc|c|ccc|c} 
& \multicolumn{4}{|c|}{ pre-filtering } & \multicolumn{4}{c}{ post-filtering } \\
\hline & \multicolumn{3}{|c|}{$\mathbf{T}_{\mathbf{D}}(\mathbf{m s})$} & & & $\mathbf{T}_{\mathbf{D}}(\mathbf{m s})$ & & \\
mean \pm SD & IQR & \# curves & median & mean \pm SD & IQR & \# curves \\
\hline PRR-MTBR-R' & 2.24 & $2.52 \pm 1.41$ & 0.99 & 394 & 2.02 & $2.06 \pm 0.42$ & 0.63 & 304 \\
$\operatorname{tau}_{2 \mathrm{~N}}$ & 1.35 & $1.43 \pm 0.34$ & 0.29 & 419 & 1.29 & $1.31 \pm 0.12$ & 0.23 & 327 \\
$\operatorname{tau}_{1 \mathrm{~N}}$ & 1.55 & $1.73 \pm 1.31$ & 0.40 & 424 & 1.50 & $1.51 \pm 0.20$ & 0.34 & 383 \\
$\operatorname{tau}_{0 \mathrm{~N}}$ & 1.61 & $1.80 \pm 0.86$ & 0.47 & 434 & 1.55 & $1.57 \pm 0.25$ & 0.36 & 378
\end{tabular}

Statistics of the diffusion times from select tau constructs incubated with $10 \mu \mathrm{M}$ tubulin without and with our filtering algorithm. IQR and SD stand for interquartile range and standard deviation respectively. See SI Appendix for details. 
Table S4. Tau-mediated Polymerization

\begin{tabular}{c|c|c} 
& & \\
construct & $\mathbf{t}_{1 / 2}(\mathbf{s})$ & tau : tubulin \\
\hline \hline PRR-MTBR-R' & $52 \pm 7$ & $1: 2$ \\
$\operatorname{tau}_{2 \mathrm{~N}}$ & $137 \pm 9$ & $1: 2$ \\
$\operatorname{tau}_{1 \mathrm{~N}}$ & $88 \pm 13$ & $1: 2$ \\
$\operatorname{tau}_{\mathrm{ON}}$ & $76 \pm 10$ & $1: 2$ \\
$\mathrm{PRR}$ & $96 \pm 55$ & $1: 1$
\end{tabular}

Polymerization half-times $\left(\mathrm{t}_{1 / 2}\right)$ for tau constructs shown in Figs. 2 and 5. Values listed for $\mathrm{t}_{1 / 2}$ are mean \pm $\mathrm{SD}$ for $\mathrm{n} \geq 3$ independent measurements. The tau:tubulin ratio used in each experiment is also listed. 
Table S5. Summary of FCS diffusion times

\begin{tabular}{|c|c|c|c|}
\hline & - tubulin & + tubulin & $\begin{aligned} & T_{\text {norm }} \\
+ & \text { tubulin }\end{aligned}$ \\
\hline $\operatorname{tau}_{2 \mathrm{~N}}$ & $0.80 \pm 0.03$ & $1.54 \pm 0.09$ & $0.92 \pm 0.09$ \\
\hline $\operatorname{tau}_{1 \mathrm{~N}}$ & $0.78 \pm 0.03$ & $1.53 \pm 0.14$ & $0.96 \pm 0.14$ \\
\hline $\operatorname{tau}_{\mathrm{ON}}$ & $0.79 \pm 0.02$ & $1.57 \pm 0.12$ & $1.00 \pm 0.12$ \\
\hline $\operatorname{tau}_{\Delta^{N}}$ & $0.78 \pm 0.02$ & $2.71 \pm 0.73$ & $2.48 \pm 0.73$ \\
\hline $\operatorname{tau}_{\Delta \mathrm{c}}$ & $0.79 \pm 0.05$ & $1.51 \pm 0.15$ & $0.92 \pm 0.15$ \\
\hline $2 \mathrm{~N}$ & $0.53 \pm 0.07$ & $0.53 \pm 0.06$ & $-0.01 \pm 0.06$ \\
\hline PRR & $0.47 \pm 0.01$ & $0.82 \pm 0.03$ & $0.75 \pm 0.03$ \\
\hline MTBR & $0.50 \pm 0.02$ & $0.52 \pm 0.01$ & $0.05 \pm 0.01$ \\
\hline MTBR-R' & $0.52 \pm 0.01$ & $0.76 \pm 0.05$ & $0.44 \pm 0.05$ \\
\hline PRR-MTBR-R' & $0.72 \pm 0.01$ & $2.67 \pm 0.63$ & $2.72 \pm 0.63$ \\
\hline PRR-MTBR-R' * & $0.73 \pm 0.01$ & $2.41 \pm 0.99$ & $2.30 \pm 0.99$ \\
\hline PRR-MTBR & $0.70 \pm 0.01$ & $2.61 \pm 0.26$ & $2.25 \pm 0.26$ \\
\hline 2N-PRR & $0.62 \pm 0.01$ & $0.76 \pm 0.01$ & $0.23 \pm 0.01$ \\
\hline $1 \mathrm{~N}-\mathrm{PRR}$ & $0.57 \pm 0.01$ & $0.77 \pm 0.04$ & $0.35 \pm 0.04$ \\
\hline ON-PRR & $0.51 \pm 0.02$ & $0.71 \pm 0.01$ & $0.40 \pm 0.01$ \\
\hline 2N-MTBR-R' & $0.72 \pm 0.01$ & $0.72 \pm 0.02$ & $-0.01 \pm 0.02$ \\
\hline $\mathrm{P} 1$ & $0.32 \pm 0.01$ & $0.37 \pm 0.01$ & $0.16 \pm 0.01$ \\
\hline P2 & $0.32 \pm 0.02$ & $0.39 \pm 0.02$ & $0.21 \pm 0.02$ \\
\hline P2-MTBR & $0.60 \pm 0.01$ & $1.37 \pm 0.18$ & $1.27 \pm 0.18$ \\
\hline P2-MTBR * & $0.67 \pm 0.06$ & $0.89 \pm 0.13$ & $0.33 \pm 0.13$ \\
\hline
\end{tabular}

Diffusion times $\left(\tau_{\mathrm{D}}\right)$ of tau constructs in the absence and presence of $10 \mu \mathrm{M}$ tubulin. Values are mean $\pm \mathrm{SD}$ for $\mathrm{n} \geq 3$ independent measurements. (*) Indicates measurements with $300 \mathrm{mM} \mathrm{KCl}$. The $\tau_{\text {norm }}$ is calculated as described in the SI Appendix. 
Table S6. Summary of CPM from FCS measurements

\begin{tabular}{|c|c|c|c|}
\hline & \multicolumn{2}{|c|}{ CPM (kHz) } & \multirow{2}{*}{$\begin{array}{l}\mathrm{CPM}_{\text {norm }} \\
+ \text { tubulin }\end{array}$} \\
\hline & - tubulin & + tubulin & \\
\hline $\operatorname{tau}_{2 \mathrm{~N}}$ & $11 \pm 4$ & $15 \pm 2$ & 1.3 \\
\hline $\operatorname{tau}_{1 \mathrm{~N}}$ & $12 \pm 1$ & $15 \pm 2$ & 1.3 \\
\hline $\operatorname{tau}_{0 \mathrm{~N}}$ & $12 \pm 1$ & $18 \pm 2$ & 1.5 \\
\hline $\operatorname{tau}_{\Delta \mathrm{N}}$ & $18 \pm 2$ & $35 \pm 8$ & 1.9 \\
\hline $\operatorname{tau}_{\Delta^{c}}$ & $9 \pm 1$ & $16 \pm 3$ & 1.7 \\
\hline $2 \mathrm{~N}$ & $7 \pm 1$ & $8 \pm 1$ & 1.1 \\
\hline PRR & $9 \pm 1$ & $9 \pm 1$ & 1.0 \\
\hline MTBR & $10 \pm 1$ & $10 \pm 1$ & 1.0 \\
\hline MTBR-R' & $9 \pm 1$ & $9 \pm 1$ & 1.0 \\
\hline PRR-MTBR-R' & $9 \pm 2$ & $26 \pm 9$ & 2.9 \\
\hline PRR-MTBR-R' * & $10 \pm 1$ & $20 \pm 6$ & 2.0 \\
\hline PRR-MTBR & $11 \pm 1$ & $22 \pm 2$ & 2.0 \\
\hline 2N-PRR & $8 \pm 1$ & $8 \pm 1$ & 1.0 \\
\hline $1 \mathrm{~N}-\mathrm{PRR}$ & $8 \pm 1$ & $7 \pm 1$ & 0.9 \\
\hline ON-PRR & $8 \pm 1$ & $7 \pm 1$ & 0.9 \\
\hline 2N-MTBR-R' & $8 \pm 1$ & $9 \pm 1$ & 1.0 \\
\hline $\mathrm{P} 1$ & $10 \pm 1$ & $10 \pm 1$ & 1.0 \\
\hline P2 & $11 \pm 2$ & $12 \pm 2$ & 1.0 \\
\hline P2-MTBR & $10 \pm 1$ & $13 \pm 2$ & 1.3 \\
\hline P2-MTBR * & $11 \pm 2$ & $10 \pm 1$ & 0.9 \\
\hline
\end{tabular}

$\mathrm{CPM}$ of tau constructs in the absence and presence of $10 \mu \mathrm{M}$ tubulin. Values given are mean $\pm \mathrm{SD}$ for $\mathrm{n} \geq 3$ independent measurements. (*) Indicates measurements with $300 \mathrm{mM} \mathrm{KCl}$. $\mathrm{CPM}_{\text {norm }}$ is the average CPM of labeled tau in the presence of tubulin divided by CPM of labeled tau without tubulin. 


\section{SI References}

1. Castoldi M, Popov AV (2003) Purification of brain tubulin through two cycles of polymerizationdepolymerization in a high-molarity buffer. Protein Expr Purif 32:83-88.

2. Melo AM, et al. (2016) A functional role for intrinsic disorder in the tau-tubulin complex. Proc Natl Acad Sci USA 113:14336-14341.

3. Li X-H, Rhoades E (2017) Heterogeneous tau-tubulin complexes accelerate microtubule polymerization. Biophys $J$ 112:2567-2574.

4. Elbaum-Garfinkle S, Cobb G, Compton JT, Li X-H, Rhoades E (2014) Tau mutants bind tubulin heterodimers with enhanced affinity. Proc Natl Acad Sci USA 111:6311-6316.

5. Mukrasch MD, et al. (2007) The "jaws" of the tau-microtubule interaction. J Biol Chem 282:1223012239.

6. Li X-H, Culver JA, Rhoades E (2015) Tau binds to multiple tubulin dimers with helical structure. $J$ Am Chem Soc 137:9218-9221.

7. Kapanidis AN, et al. (2004) Fluorescence-aided molecule sorting: Analysis of structure and interactions by alternating-laser excitation of single molecules. Proc Natl Acad Sci USA 101:89368941.

8. Metskas LA, Rhoades E (2015) Conformation and dynamics of the troponin i c-terminal domain: Combining single-molecule and computational approaches for a disordered protein region. $\mathrm{J} \mathrm{Am}$ Chem Soc 137:11962-11969.

9. Trexler AJ, Rhoades E (2010) Single molecule characterization of $\alpha$-synuclein in aggregation-prone states. Biophys J 99:3048-3055.

10. O'Brien EP, Morrison G, Brooks BR, Thirumalai D (2009) How accurate are polymer models in the analysis of förster resonance energy transfer experiments on proteins? The Journal of Chemical Physics 130:124903.

11. Kohn JE, et al. (2004) Random-coil behavior and the dimensions of chemically unfolded proteins. Proc Natl Acad Sci USA 101:12491-12496. 